\title{
Special functions for the study of economic dynamics: The case of the Lucas-Uzawa model*
}

\author{
R. Boucekkine ${ }^{\dagger} \quad$ J. R. Ruiz-Tamarit ${ }^{\ddagger}$
}

October, 2004

\begin{abstract}
The special functions are intensively used in mathematical physics to solve differential systems. We argue that their use should be most useful in economic dynamics, notably in the assessment of the transition dynamics of endogenous growth models. We illustrate our argument on the Lucas-Uzawa model, which we solve by the means of Gaussian hypergeometric functions. We show how the use of Gaussian hypergeometric functions allows for an explicit representation of the equilibrium dynamics of the variables in level. In contrast to the preexisting approaches, our method is global and does not rely on dimension reduction.
\end{abstract}

Keywords: Special functions, hypergeometric functions, optimal control, LucasUzawa model, economic dynamics

JEL classification: C61, C62, O41.

*We are indebted to Vadim Kuznetsov, Alphonse Magnus, Nico Temme, Benteng Zou for useful suggestions and comments. R. Boucekkine acknowledges the support of the Belgian research programmes PAI P4/01 and ARC 03/08-302. J. R. Ruiz-Tamarit acknowledges the financial support from the Spanish CICYT, Project SEC2000-0260, and the Grant PR2003-0107 from the Secretaría de Estado de Educación y Universidades, Spanish MECD.

${ }^{\dagger}$ Corresponding author. Department of Economics and CORE, Université Catholique de Louvain, Place Montesquieu 3, B-1348 Louvain-la-Neuve (Belgium). E-mail: boucekkine@ires.ucl.ac.be

${ }^{\ddagger}$ Department of Economics, Universitat de València (Spain) and Université catholique de Louvain. E-mail: ramon.ruiz@uv.es 


\section{Introduction}

Special functions refer to some specific functions with special characteristics, typically used in mathematical physics and computational mathematics. Prominent examples include the Gamma function, the Beta function, the Bessel function and the more general, hypergeometric functions. There exist some excellent reviews on the properties and uses of such functions, among them Luke (1969), Abramowitz and Stegun (1972), and more recently Temme (1996) and Andrews, Askey and Roy (1999). The area of special functions is, by no way, a new research area: it traces back at least to Euler and Gauss. Among their multiple uses, the resolution of differential equations and systems is not the least important nor the least historically recognized. This is the case of Gauss hypergeometric functions, which allowed to solve nicely the so-called hypergeometric differential equation (Kummer, 1836, or Goursat, 1881). We shall also use this class of functions in our study. Indeed, we argue in this paper that researchers in economic dynamics should use much more intensively these tools, which can be decisive if one aims at getting beyond the typical computational and/or local approaches. More specifically, we argue that such functions might be most useful in the assessment of the transition dynamics and asymptotics of endogenous growth models.

Traditionally economists have studied non-linear dynamic systems, arising from optimal control problems, using qualitative techniques (like phase diagrams), complemented with a certain quantitative analysis mostly based on linearization around steady states. 1 However, both approaches require the existence of isolated steady states. Moreover, for the linearization to be safe (in terms of the -local- asymptotic stability diagnosis for the original nonlinear model, notably), one needs to ensure some regularity conditions that are typically stated in the well-known Hartman-Grobman theorem. It turns out that such a fundamental theorem sets a serious limitation on the use of the traditional techniques in economic dynamics to investigate the stability properties of endogenous growth models of the non-AK type. ${ }^{2}$

\footnotetext{
${ }^{1}$ As an alternative procedure, the so called Linear-Quadratic approximation proceeds by "quadrifying" the objective functional in the optimization problem to have the optimal decision rules directly in linear form. After having substituted the non-linear constraints into the return function, the local behavior of the model around the steady state is driven by a linear dynamic system. In this case, a laborious arithmetic is needed in the quadrification process. The linear systems may be solved explicitly in a closed analytical form.

${ }^{2}$ It goes without saying that we are not concerned with the class of AK models such like the labequipment model of Rivera-Batiz and Romer (1991), which are mathematically trivial in that they admit immediate closed form solutions and no transition dynamics.
} 
In effect, it is well known that the Hartman-Grobman theorem does not hold when the determinant of the Jacobian matrix evaluated at the steady state equilibrium becomes zero, when a steady state equilibrium does not exist or when there is a continuum of them. In such cases, the linear approximation cannot be used to study the dynamic behavior of the original nonlinear system, even locally. ${ }^{3}$ Unfortunately, a striking property of endogenous growth models is the indeterminacy of the steady state levels of the considered (trended) variables, which disqualifies linearization de facto.

In this context, the dimension reduction strategy has been widely applied. People define new variables as ratios between the variables of the original system -states, costates and controls- in such a way that they transform the original system into another system with a lower dimension. Then, they study this transformed system and look for steady states which will be interpreted as balanced growth paths for the original system. Obviously, the complete knowledge of the dynamics associated with the reduced model is generally not sufficient to be fully conclusive about the dynamic properties of the original model. By construction, the reduction of dimension entails a loss of information which may lead to mistakes and miss-interpretations. In any case, while the reduction technique is definitely useful to get a highly informative picture of the dynamics at work in the models, it cannot deliver more than what it can, and in particular, it cannot serve to fully characterize the dynamics of the original variables in level. The purpose of this paper is to argue that conveniently chosen special functions could be the solution to this very known problem.

To illustrate our point, we choose the Lucas-Uzawa model, one of the most celebrated endogenous growth model, which has some interesting properties. It is not of the AK type, and moreover, being a two-sector model, it gives rise to a sophisticated dynamic system with two controls and two state variables. Last but not least, because it is mathematically appealing, it has been studied by many authors, using different approaches, therefore allowing for a stimulating methodological discussion. The seminal model is Lucas (1988). Barro and Sala-i-Martin (1995), chapter 5, have analyzed such a model using numerical simulations and qualitative reasoning. However, the purely computational approach followed in the above mentioned articles has led certain authors to question its contributions. For example, Xie (1994) describes the transitional dynamics and stability analysis in Mulligan and Sala-i-Martin (1993) as non-transparent. More broadly speak-

\footnotetext{
${ }^{3}$ To be a bit more comprehensive, by the same Hartman-Grobman theorem, linearization is no longer safe also if an isolated steady state exists provided it is only stable but not asymptotically stable in the sense of Lyapunov.
} 
ing, there is an obvious need to complement this computational and qualitative literature with analytical propositions and theoretical proofs and, indeed, some few contributions have came out taking exactly this approach. In addition to Xie (1994), Caballé and Santos (1993), Benhabib and Perli (1994) and Bond, Wang and Yip (1996) are by far the most comprehensive and rigorous. While Xie proposes an analytical solution of the Lucas model in a particular parametric case, the three other contributions provide a deep local stability analysis in some general cases.

In the original Lucas 1988 paper, the author only studied the properties of a particular solution trajectory which corresponds to the system's balanced growth path, leaving in the dark the remaining off-steady-state behavior. An important stream of literature came later to assess the initial conjectures of Lucas relative to the transitional dynamics of the system. On the one hand, Mulligan and Sala-i-Martín (1993) analyzed the short run dynamics by means of the time elimination method. Their procedure builds upon a fundamental transformation of the 4-dimension original system by defining control-like and state-like variables. Then, they characterize a stationary point and its stable manifold in the 3-dimension system which derives from the substitution of such auxiliary variables. Time elimination method then allows for a further transformation of the boundary value problem into a two-dimensional initial value problem. This is done by manipulating the policy functions and eliminating time. The resulting two-dimensional dynamic system is finally solved using standard numerical techniques.

Benhabib and Perli (1994) take a different approach. After writing the original dynamic system in terms of states and controls, they reduce the dimension of the system by defining the same state-like and control-like variables as Mulligan and Sala-i-Martín. Then, they study the existence, uniqueness and multiplicity of steady states in such a reduced system, which are automatically identified as balanced growth paths in the context of the original system. They also determine fundamental parameter constraints which guarantee interior steady states and use transversality conditions only to check that these stationary trajectories satisfy the non-explosivity conditions. Ultimately, they perform a local dynamic stability study of the reduced dynamic system by evaluating its Jacobian matrix and characterizing the solution space by looking at the signs of the different eigenvalues. The system shows a unique solution of the saddle path type but, in some cases, it allows for multiplicity of solutions because of the two free initial conditions and a unique positive eigenvalue. Caballé and Santos (1993) is another very careful inspection into the dynamic properties of the Lucas model. Removing the Lucas externality, which disables 
multiplicity of steady states, they finely studied the transition dynamics of the model by distinguishing several relevant parametric cases. We shall show that this parametric characterization of Caballé and Santos is also most relevant in our approach. Nonetheless, just like Benhabib and Perli, their mathematical apparatus is still local and since their main convergence results focus on the ratio human to physical capital, they also resort to dimension reduction.

Indeed, the three previous contributions are based on a dimension reduction device, and they typically come out with a comprehensive dynamic analysis of some useful economic ratios. It could be the case that these ratios are enough to study some important economic problems. However, it is also out of question that being able to characterize the dynamics of the original variables (typically, the levels of per capita consumption, output, physical capital and human capital stock...etc...) is a crucial challenge because these dynamics are fundamental to get a complete picture of the economic system. One way to achieve this task is to develop analytical methods. This is done by Xie (1994) who develops a method allowing to explicitly compute the equilibrium dynamics. Unfortunately, the method only works under the very strong assumption that the inverse of the intertemporal elasticity of substitution for consumption in utility function equals the elasticity of output with respect to physical capital in goods sector. Clearly this assumption limits drastically the scope of Xie's results, but the whole approach is interesting in that it shows a nicely articulated effort to go beyond the typical computational and/or local strategies. Our paper follows this analytical line. We show how the Gaussian hypergeometric functions can be used to construct explicit representations of the dynamics of the variables in level, allowing for a global analysis and without restricting conditions on the parameters. ${ }^{4}$ Among the numerous new results allowed by the method, one could mention the non-monotonicity of most variables in level, a property that may be "hidden" by the monotonicity of the ratios of variables usually considered in the related literature.

The next sections are organized as follows: Section 2 recalls the Lucas model without externality using the same notation as Caballé and Santos (1993), giving the first order conditions and specifying the non-linear dynamic system which drives the economy in equilibrium. Section 3 is designed first to briefly introduce to Gauss hypergeometric functions and, more importantly, to show how they can be actually used to obtain explicit representations of the equilibrium dynamics of physical and human capital in the LucasUzawa model. Section 4 connects the latter result with the remaining optimality criteria

\footnotetext{
${ }^{4}$ except the usual conditions in endogenous growth theory, which are required to meet the transversality conditions and to ensure the positivity of certain economic variables.
} 
(mainly the transversality conditions) and characterizes the optimal equilibrium paths in terms of existence, uniqueness, positivity and monotonicity. Section 5 is the economic section of the paper: we study some economic properties of some well defined balanced growth paths, and we present some numerical experiments for different parameterizations of the model, following Caballé and Santos (1993). Section 6 concludes.

\section{The Uzawa-Lucas model}

Consider the Uzawa-Lucas two-sector model of endogenous growth. The economy is closed with competitive markets and populated with many identical, rational agents, facing up to the problem that consists in choosing the controls $c(t)$ and $u(t) \forall t \geq t_{0}$ which solve the dynamic optimization problem

$$
\max \int_{0}^{\infty} \frac{c(t)^{1-\sigma}-1}{1-\sigma} N(t) e^{-\rho t} d t
$$

subject to

$$
\begin{gathered}
\dot{K}(t)=A K(t)^{\beta}(u(t) N(t) h(t))^{1-\beta}-\pi K(t)-N(t) c(t), \\
\dot{h}(t)=\delta(1-u(t)) h(t)-\theta h(t), \\
K(0)=K_{0}, \quad h(0)=h_{0}, \quad N(0)=N_{0}, \\
c(t) \geqslant 0, \quad u(t) \in[0,1], \quad K(t) \geqslant 0, \quad h(t) \geqslant 0 .
\end{gathered}
$$

Here $c(t)$ is the stream of real per capita consumption of a single good. The instantaneous utility function is a CRRA function where $\sigma^{-1}>0$ represents the constant elasticity of intertemporal substitution. Population at time $t$ is $N(t)$, which is assumed to grow at a constant exogenously given rate $n$. Parameter $\rho$ is the rate of time preference or discount rate. We assume $\rho>n$ In this model $h(t)$ is the human capital level, or the skill level, of a representative worker while $u(t)$ is the fraction of non-leisure time devoted to goods production. The output, $Y(t)$, which may be allocated to consumption or to physical capital accumulation depends on the capital stock, $K(t)$, and the effective work force, $u(t) N(t) h(t)$. Parameter $\beta$ is the elasticity of output with respect to physical capital. The efficiency parameter $A$ represents the constant technological level in the goods sector of this economy. It is assumed that the growth of human capital do not depend on the physical capital stock. It depends on the effort devoted to the accumulation of human capital, $1-u(t)$, as well as on the already attained human capital stock. The efficiency 
parameter $\delta$ represents the constant technological level in the educational sector. It also represents the maximal gross rate of growth for $h(t)$ attainable when all effort is devoted to human capital accumulation. Technology in goods sector shows constant return to scale over private internal factors. Technology in educational sector is linear. Both physical and human capital depreciate at constant rates, which are $\pi \geqslant 0$ and $\theta \geqslant 0$, respectively. We shall also assume that $\delta+n>\theta+\rho$ for positive (long run) growth to arise, as it will be transparent in Section 4. Note that this assumption also implies that $\delta+n+\pi-\theta>0 .{ }^{5}$ The current value Hamiltonian associated with the previous intertemporal optimization problem is

$$
\begin{gathered}
H^{c}\left(K, h, \vartheta_{1}, \vartheta_{2}, c, u ; A, \sigma, \beta, \delta, \pi, \theta,\{N(t): t \geq 0\}\right)= \\
=\frac{c^{1-\sigma}-1}{1-\sigma} N+\vartheta_{1}\left[A K^{\beta}(u N h)^{1-\beta}-\pi K-N c\right]+\vartheta_{2}[\delta(1-u) h-\theta h]
\end{gathered}
$$

where $\vartheta_{1}$ and $\vartheta_{2}$ are the co-state variables for $K$ and $h$, respectively.

The first order necessary conditions are

$$
\begin{gathered}
c^{-\sigma}=\vartheta_{1}, \\
\vartheta_{1}(1-\beta) A K^{\beta}(u N h)^{-\beta} N=\vartheta_{2} \delta,
\end{gathered}
$$

the Euler equations

$$
\begin{gathered}
\dot{\vartheta}_{1}=(\rho+\pi) \vartheta_{1}-\vartheta_{1} \beta A K^{\beta-1}(u N h)^{1-\beta}, \\
\dot{\vartheta}_{2}=(\rho+\theta) \vartheta_{2}-\vartheta_{1}(1-\beta) A K^{\beta}(u N)^{1-\beta} h^{-\beta}-\vartheta_{2} \delta(1-u),
\end{gathered}
$$

the dynamic constraints

$$
\begin{gathered}
\stackrel{\bullet}{K}=A K^{\beta}(u N h)^{1-\beta}-\pi K-N c, \\
\dot{h}=\delta(1-u) h-\theta h,
\end{gathered}
$$

the boundary conditions $K_{0}, h_{0}$, and the transversality conditions

$$
\begin{aligned}
& \lim _{t \rightarrow \infty} \vartheta_{1} K \exp \{-\rho t\}=0, \\
& \lim _{t \rightarrow \infty} \vartheta_{2} h \exp \{-\rho t\}=0 .
\end{aligned}
$$

Notice that by $(3), \vartheta_{1}(t)$ cannot be equal to 0 at any finite date $t$ because this would require that consumption is infinite at a finite date, which violates the resource constraint of the economy. Then, according to $(4), \vartheta_{2}(t) \neq 0$ at a finite $t$, provided the economy starts

\footnotetext{
${ }^{5}$ The latter expression will appear recurrently along this paper.
} 
with finite and strictly positive endowments of physical and human capital, implying also finite and strictly positive output levels at any finite date.

On the margin, goods and time must be equally valuable in their two uses: consumption and physical capital accumulation, and production and human capital accumulation, respectively.

From (3) and (4) we get the control functions

$$
\begin{gathered}
c=\vartheta_{1}^{-\frac{1}{\sigma}} \\
u=\left(\frac{(1-\beta) A}{\delta}\right)^{\frac{1}{\beta}}\left(\frac{\vartheta_{1}}{\vartheta_{2}}\right)^{\frac{1}{\beta}} \frac{K}{h} N^{\frac{1-\beta}{\beta}} .
\end{gathered}
$$

After substituting the above expressions into equations (5)-(8), we obtain

$$
\begin{gathered}
\dot{\vartheta}_{2}=-(\delta-\rho-\theta) \vartheta_{2} \\
\dot{\vartheta}_{1}=(\rho+\pi) \vartheta_{1}-\psi_{1}(t) \vartheta_{1}^{\frac{1}{\beta}} \\
\dot{K}=\psi_{2}(t) K-\psi_{3}(t) \\
\dot{h}=(\delta-\theta) h-\psi_{4}(t)
\end{gathered}
$$

where

$$
\begin{gathered}
\psi_{1}(t)=\beta A\left(\frac{(1-\beta) A}{\delta}\right)^{\frac{1-\beta}{\beta}} N^{\frac{1-\beta}{\beta}} \vartheta_{2}^{-\frac{1-\beta}{\beta}}, \\
\psi_{2}(t)=A\left(\frac{(1-\beta) A}{\delta}\right)^{\frac{1-\beta}{\beta}} N^{\frac{1-\beta}{\beta}}\left(\frac{\vartheta_{1}}{\vartheta_{2}}\right)^{\frac{1-\beta}{\beta}}-\pi, \\
\psi_{3}(t)=N \vartheta_{1}^{-\frac{1}{\sigma}}, \\
\psi_{4}(t)=\delta\left(\frac{(1-\beta) A}{\delta}\right)^{\frac{1}{\beta}} N^{\frac{1-\beta}{\beta}}\left(\frac{\vartheta_{1}}{\vartheta_{2}}\right)^{\frac{1}{\beta}} K .
\end{gathered}
$$

These equations, together with the initial conditions, $K_{0}$ and $h_{0}$, and the transversality conditions (9) and (10) constitute the dynamic system which drives the economy over time. This dynamic system can be recursively solved in closed form. We don't need to transform this original modified Hamiltonian dynamic system by reducing its dimension.

\section{$3 \quad$ Analytical solution using gaussian hypergeometric functions}

We first start with a short overview of hypergeometric functions, putting forward their main algebraic properties. 


\subsection{A quick overview of Gauss hypergeometric functions}

The Gauss hypergeometric function belongs to the general class of hypergeometric functions, studied in detail in the now classical textbook of Abramowitz and Stegun (1972) (see also the more recent textbook of Temme, 1996). As mentioned in the introduction section, most applications of hypergeometric functions are so far in the field of mathematical physics. Abadir (1999) has already pointed out the potential interest of these functions for economists, but there is to our knowledge no application so far to growth models. We shall present here a very short overview of the Gauss hypergeometric function, with a special emphasis on the tools which will be used along this paper.

Definition 1 The Gauss hypergeometric function, ${ }_{2} F_{1}(a, b, c ; z)$, with complex arguments $a, b, c$ and $z$, is given by the series:

$$
{ }_{2} F_{1}(a, b, c ; z)=\sum_{n=0}^{\infty} \frac{(a)_{n}(b)_{n}}{(c)_{n}} \frac{z^{n}}{n !},
$$

where $(x)_{n}$ is the so-called Pochhammer symbol, defined by:

$$
(x)_{n}=\frac{\Gamma(x+n)}{\Gamma(x)}
$$

where $\Gamma($.$) is the special function Gamma.$

One of the main properties of the Gauss hypergeometric function is that its circle of convergence is the unit circle, that it is the series is uniformly converging when $|z|<1$ (Abramowitz and Stegun, page 556, section 15.1.1). When $|z|=1$, the series is either divergent if $\operatorname{Re}(c-a-b) \leq 1$, or absolutely convergent whenever $\operatorname{Re}(c-a-b)>0$, or conditionally convergent if $-1<\operatorname{Re}(c-a-b) \leq 0$, where $\operatorname{Re}(z)$ is the real part of $z$. Another interesting property of the Gauss hypergeometric function is that it degenerates into a polynomial of degree $n$ in $z$ if $a$ or $b$ is equal to $-n$, where $n$ is a positive integer, including $n=0$ (see again Abramowitz and Stegun, page 556, Section 15.1.1). This property will be used in Section 4.2. Because it involves the Gamma function which is not analytic for negative integers, the Gauss hypergeometric function is not defined when $c=-m$, for $m=0,1,2,3$. , provided $a$ or $b$ is not a negative integer.

Is there a way to define the Gauss hypergeometric function outside its unit circle? This is a very important issue that has been the subject of many recent and less recent developments (see for example Becken and Schmelcher, 2000, and Temme, 2003). Indeed, there are many continuation formulas of the Gauss hypergeometric function outside the unit circle (see Abramowitz and Stegun, page 559, formulas 15.3.3. to 15.3.9 for example). For example, 
for $|z|>1$, one can use formula 15.3.7 in Abramowitz and Stegun, page 559, or formula (9) in Temme (2003), page 442:

$$
\begin{gathered}
{ }_{2} F_{1}(a, b, c ; z)=\frac{\Gamma(c) \Gamma(b-a)}{\Gamma(b) \Gamma(c-a)}(-z)^{-a}{ }_{2} F_{1}\left(a, 1-c+a, 1-b+a ; \frac{1}{z}\right) \\
+\frac{\Gamma(c) \Gamma(a-b)}{\Gamma(a) \Gamma(c-b)}(-z)^{-b}{ }_{2} F_{1}\left(b, 1-c+b, 1-a+b ; \frac{1}{z}\right)
\end{gathered}
$$

which is valid as long as $|\arg (-z)|<\pi$, using Definition 1 to compute the values of the Gauss hypergeometric functions appearing on the right hand side of the formula just above. The continuation formulas show that the Gauss hypergeometric function can be defined as an analytic function in the whole complex plane, cut along the real axis from 1 to $\infty$. However, because of this branch cut, one has to choose the correct continuation formula. The value of the Gauss hypergeometric function for $|z|>1$ depends on the way you approach this line in the complex plane.

However, the most practical continuation formulas (for reasons which will be crystal clear along this paper) consist in the integral representations of the Gauss hypergeometric function. We shall use the following, commonly known as the Euler integral representation:

$$
{ }_{2} F_{1}(a, b, c ; z)=\frac{\Gamma(c)}{\Gamma(b) \Gamma(c-b)} \int_{0}^{1} t^{b-1}(1-t)^{c-b-1}(1-t z)^{-a} d t
$$

when $\operatorname{Re}(c)>\operatorname{Re}(b)>0$ (see Abramowitz and Stegun, 15.3.1, page 558). Notice that by construction and provided the parameter conditions written just above are met, the Gauss hypergeometric function is a one valued analytic function in the whole plan cut along the real axis from 1 to $\infty$. As one may guess, the Euler representation is most practical for signing, differentiating and almost for all the typical algebraic tasks. We will show that fortunately our solution paths involve such hypergeometric functions with the easiest integral representation.

In case we don't have the Euler presentation, we still have some easy differentiation rules, which can be quite easily demonstrated from the original infinite expansions representations of the functions. In Abramowitz and Stegun, page 557, formula 15.2.1 gives the simple differentiation rule:

$$
\frac{d}{d z}{ }_{2} F_{1}(a, b, c ; z)=\frac{a b}{c}{ }_{2} F_{1}(a+1, b+1, c+1 ; z) .
$$

Another very useful rule, which is used in the next section is derived from Abramowitz and Stegun, page 557, formula 15.2.3:

$$
\frac{d}{d z} z_{2}^{s} F_{1}(s, b, c ; z)=s z^{s-1}{ }_{2} F_{1}(s+1, b, c ; z) .
$$




\subsection{Gauss hypergeometric representations of the solution paths}

The complete closed-form solution for the variables appearing in the dynamic system (13)-(16), as well as for the controls of the model, may be found sequentially according the following procedure. First, taking (13) and integrating we get

$$
\vartheta_{2}=\vartheta_{2}(0) \exp \{-(\delta-\rho-\theta) t\}
$$

where $\vartheta_{2}(0)$ has still to be determined. Second, substitute (21) and the exogenous population growth process $N=N_{0} \exp \{n t\}$ in (17). Then, the standard Bernoulli's differential equation in $\vartheta_{1}(14)$ has the following general solution

$$
\begin{gathered}
\vartheta_{1}=\left(\frac{\delta+n+\pi-\theta}{\epsilon}\right)^{\frac{\beta}{1-\beta}} \vartheta_{2}(0) \exp \{(\rho+\pi) t\} \\
\cdot\left[-1+\exp \left\{\frac{(1-\beta)(\delta+n+\pi-\theta)}{\beta} t\right\}+\frac{\delta+n+\pi-\theta}{\epsilon}\left(\frac{\vartheta_{1}(0)}{\vartheta_{2}(0)}\right)^{-\frac{1-\beta}{\beta}}\right]^{-\frac{\beta}{1-\beta}}
\end{gathered}
$$

where $\vartheta_{1}(0)$ and $\vartheta_{2}(0)$ are still undetermined and $\epsilon=\beta A\left(\frac{(1-\beta) A N_{0}}{\delta}\right)^{\frac{1-\beta}{\beta}}>0$.

Now, we substitute (21) and (22) in (18) and (19) getting

$$
\psi_{2}(t)=\frac{1}{\frac{\beta}{\delta+n+\pi-\theta}+\left(\frac{\beta}{\epsilon}\left(\frac{\vartheta_{1}(0)}{\vartheta_{2}(0)}\right)^{-\frac{1-\beta}{\beta}}-\frac{\beta}{\delta+n+\pi-\theta}\right) \exp \left\{-\frac{(1-\beta)(\delta+n+\pi-\theta)}{\beta} t\right\}}-\pi
$$

and

$$
\begin{gathered}
\psi_{3}(t)=\left(\frac{\epsilon}{\delta+n+\pi-\theta}\right)^{\frac{\beta}{\sigma(1-\beta)}} N_{0}\left(\vartheta_{2}(0)\right)^{-\frac{1}{\sigma}} \exp \left\{-\frac{\rho+\pi-n \sigma}{\sigma} t\right\} \\
\cdot\left[-1+\exp \left\{\frac{(1-\beta)(\delta+n+\pi-\theta)}{\beta} t\right\}+\frac{\delta+n+\pi-\theta}{\epsilon}\left(\frac{\vartheta_{1}(0)}{\vartheta_{2}(0)}\right)^{-\frac{1-\beta}{\beta}}\right]^{\frac{\beta}{\sigma(1-\beta)}} .
\end{gathered}
$$

The general solution to the linear differential equation in $K$ with time-dependent coefficients $(15)$ is

$$
K=K_{0} \exp \left\{\int_{0}^{t} \psi_{2}(s) d s\right\}-\int_{0}^{t} \psi_{3}(r) \exp \left\{\int_{r}^{t} \psi_{2}(z) d z\right\} d r
$$

or

$$
K=\frac{K_{0} e^{-\pi t}}{\left(\frac{\delta+n+\pi-\theta}{\epsilon}\left(\frac{\vartheta_{1}(0)}{\vartheta_{2}(0)}\right)^{-\frac{1-\beta}{\beta}}\right)^{\frac{1}{1-\beta}}}\left[A_{0}+\exp \left\{\frac{(1-\beta)(\delta+n+\pi-\theta)}{\beta} t\right\}\right]^{\frac{1}{1-\beta}}
$$




$$
-\frac{N_{0} e^{-\pi t}\left(\frac{\epsilon}{\delta+n+\pi-\theta}\right)^{\frac{\beta}{\sigma(1-\beta)}}}{\vartheta_{2}(0)^{\frac{1}{\sigma}}}\left[A_{0}+\exp \left\{\frac{(1-\beta)(\delta+n+\pi-\theta)}{\beta} t\right\}\right]^{\frac{1}{1-\beta}} \int_{0}^{t} Q(r) d r
$$

where

$\mathrm{Q}(\mathrm{r})=\left[A_{0} \exp \left\{-\frac{(1-\beta)(\rho+\pi-n \sigma-\pi \sigma)}{\beta-\sigma} r\right\}+\exp \left\{\frac{(1-\beta)((\delta+n+\pi-\theta)(\beta-\sigma)-\beta(\rho+\pi-n \sigma-\pi \sigma))}{\beta(\beta-\sigma)} r\right\}\right]^{\frac{\beta-\sigma}{\sigma(1-\beta)}}$, and

$$
A_{0}=\frac{\delta+n+\pi-\theta}{\epsilon}\left(\frac{\vartheta_{1}(0)}{\vartheta_{2}(0)}\right)^{-\frac{1-\beta}{\beta}}-1
$$

It remains to find an explicit form for the value of the integral in the solution trajectory for $K$. This is done using precisely the gaussian hypergeometric function representation.

Proposition 1 The integral in the capital stock expression can be computed exactly as:

$$
\int_{0}^{t} Q(r) d r=\frac{\sigma \beta\left({ }_{2} F_{1}(t) \exp \left\{\frac{(\delta+n+\pi-\theta)(\beta-\sigma)-\beta(\rho+\pi-n \sigma-\pi \sigma)}{\beta \sigma} t\right\}-{ }_{2} F_{1}(0)\right)}{(\delta+n+\pi-\theta)(\beta-\sigma)-\beta(\rho+\pi-n \sigma-\pi \sigma)},
$$

where

$$
\begin{gathered}
{ }_{2} F_{1}(t)={ }_{2} F_{1}\left(a, b, c ;\left(1-\frac{\delta+n+\pi-\theta}{\epsilon}\left(\frac{\vartheta_{1}(0)}{\vartheta_{2}(0)}\right)^{-\frac{1-\beta}{\beta}}\right) \exp \left\{-\frac{(1-\beta)(\delta+n+\pi-\theta)}{\beta} t\right\}\right), \\
{ }_{2} F_{1}(0)={ }_{2} F_{1}\left(a, b, c ; 1-\frac{\delta+n+\pi-\theta}{\epsilon}\left(\frac{\vartheta_{1}(0)}{\vartheta_{2}(0)}\right)^{-\frac{1-\beta}{\beta}}\right), \\
a=-\frac{(\delta+n+\pi-\theta)(\beta-\sigma)-\beta(\rho+\pi-n \sigma-\pi \sigma)}{\sigma(\delta+n+\pi-\theta)(1-\beta)}, \quad b=-\frac{\beta-\sigma}{\sigma(1-\beta)}, \quad c=1+a .
\end{gathered}
$$

Proof: We shall first rewrite $Q($.$) as follows: Q(r)=\left(A_{0} e^{B r}+e^{C r}\right)^{\alpha}$ with: $B=-\frac{(1-\beta)(\rho+\pi-n \sigma-\pi \sigma)}{\beta-\sigma}$, $C=\frac{(1-\beta)((\delta+n+\pi-\theta)(\beta-\sigma)-\beta(\rho+\pi-n \sigma-\pi \sigma))}{\beta(\beta-\sigma)}$, and $\alpha=\frac{\beta-\sigma}{\sigma(1-\beta)}$. We may write:

$$
Q(r)=e^{\alpha C r}\left[1+A_{0} e^{(B-C) r}\right]^{\alpha} .
$$

We shall prove the result using the Euler integral representation of Gaussian hypergeometric functions given in Section 3.1. Recall that this representation has the notable advantage (with respect to the definition of hypergeometric functions in terms of infinite expansions) that it yields an analytical function in the plane cut along the real axis from 1 to $\infty$ (while the expansions only define the hypergeometric functions in the unit disc). A proof using the expansions via the binomial theorem is given in the appendix. At first, let us use the symmetry in the arguments $a$ and $b$ of any Gaussian hypergeometric, that is: ${ }_{2} F_{1}(a, b, c ; z)={ }_{2} F_{1}(b, a, c ; z)$. If our claim is correct, we have to express the 
integral $\int_{0}^{t} Q(r) d r$ in terms of hypergeometric functions ${ }_{2} F_{1}(b, a, c ; z)$ where $c=1+a$. Therefore, we are in a situation where the involved hypergeometric functions check the conditions under which the Euler integral representation holds, that is the real part of the second argument (here $a$ ) should be positive and strictly lower than the real part of the third argument (here $c=1+a$ ). The corresponding integral representation is:

$$
\begin{gathered}
{ }_{2} F_{1}(a, b, a+1 ; z)={ }_{2} F_{1}(b, a, a+1 ; z)=\frac{\Gamma(a+1)}{\Gamma(a) \Gamma(1)} \int_{0}^{1} t^{a-1}(1-t z)^{-b} d t \\
=a \int_{0}^{1} t^{a-1}(1-t z)^{-b} d t
\end{gathered}
$$

applying again Abramowitz and Stegun, 15.3.1. and the properties $\Gamma(a+1)=a \Gamma(a)$, and $\Gamma(1)=1$. Before proceeding, notice that with our notations, $a=\frac{\alpha C}{B-C}$ and $\alpha=-b$. With the variable change $u=e^{(B-C) r}$, we have:

$$
\int_{0}^{t} Q(r) d r=\int_{0}^{t} e^{\alpha C r}\left[1+A_{0} e^{(B-C) r}\right]^{\alpha} d r=\frac{1}{B-C} \int_{1}^{e^{(B-C) t}} u^{a-1}\left(1+A_{0} u\right)^{-b} d u .
$$

We thus have already the good integrand, the rest of the proof is just straightforward but heavy algebra to get the right integration bounds. Indeed, using the integral expression obtained above, we can write:

$$
\begin{aligned}
& \int_{0}^{t} Q(r) d r=\frac{1}{B-C}\left[\int_{0}^{e^{(B-C) t}} u^{a-1}\left(1+A_{0} u\right)^{-b} d u-\int_{0}^{1} u^{a-1}\left(1+A_{0} u\right)^{-b} d u\right] \\
& \quad=\frac{a}{\alpha C}\left[e^{\alpha C t} \int_{0}^{1} u^{a-1}\left(1+A_{0} e^{(B-C) t} u\right)^{-b} d u-\int_{0}^{1} u^{a-1}\left(1+A_{0} u\right)^{-b} d u\right]
\end{aligned}
$$

which is exactly the result announced in Proposition 1, using the expressions of $A_{0}, B, C$ and $\alpha$

We now move to solve for the human capital variable. Notice that by substituting (21), (22) and the solution for $K$ in (20) we get

$$
\psi_{4}(t)=\frac{(1-\beta) \epsilon K_{0}}{\beta}\left(\frac{\vartheta_{1}(0)}{\vartheta_{2}(0)}\right)^{\frac{1}{\beta}} \frac{{ }_{2} F_{1}(t)}{{ }_{2} F_{1}(0)} \exp \left\{\frac{\delta+n-\rho-\theta}{\sigma} t\right\} .
$$

The general solution to the linear differential equation (16) is consequently

$$
h=h_{0} \exp \{(\delta-\theta) t\}-\int_{0}^{t} \psi_{4}(r) \exp \{(\delta-\theta)(t-r)\} d r
$$

or 
$h=e^{(\delta-\theta) t} \cdot\left[h_{0}-\frac{(1-\beta) \epsilon K_{0}}{{ }_{2} F_{1}(0) \beta}\left(\frac{\vartheta_{1}(0)}{\vartheta_{2}(0)}\right)^{\frac{1}{\beta}} \int_{0}^{t}{ }_{2} F_{1}(r) \exp \left\{\frac{(\delta-\theta)(1-\sigma)+n-\rho}{\sigma} r\right\} d r\right]$.

Again, we will show that the solution can be represented by the means of gaussian hypergeometric functions by finding a closed-form expression of the integral just above.

Proposition 2 The integral in the human capital stock expression can be computed exactly as:

$$
\begin{aligned}
& \int_{0}^{t}{ }_{2} F_{1}(r) \exp \left\{\frac{(\delta-\theta)(1-\sigma)+n-\rho}{\sigma} r\right\} d r \\
& =\sigma \frac{{ }_{2} \tilde{F}_{1}(t) \exp \left\{\frac{(\delta-\theta)(1-\sigma)+n-\rho}{\sigma} t\right\}-{ }_{2} \widetilde{F}_{1}(0)}{(\delta-\theta)(1-\sigma)+n-\rho}
\end{aligned}
$$

where

$$
\begin{gathered}
{ }_{2} \tilde{F}_{1}(t)={ }_{2} F_{1}\left(\tilde{a}, b, c ;\left(1-\frac{\delta+n+\pi-\theta}{\epsilon}\left(\frac{\vartheta_{1}(0)}{\vartheta_{2}(0)}\right)^{-\frac{1-\beta}{\beta}}\right) \exp \left\{-\frac{(1-\beta)(\delta+n+\pi-\theta)}{\beta} t\right\}\right), \\
{ }_{2} \tilde{F}_{1}(0)={ }_{2} F_{1}\left(\tilde{a}, b, c ; 1-\frac{\delta+n+\pi-\theta}{\epsilon}\left(\frac{\vartheta_{1}(0)}{\vartheta_{2}(0)}\right)^{-\frac{1-\beta}{\beta}}\right), \\
\tilde{a}=a-1=-\frac{(\delta+n+\pi-\theta) \beta(1-\sigma)-\beta(\rho+\pi-n \sigma-\pi \sigma)}{\sigma(\delta+n+\pi-\theta)(1-\beta)} .
\end{gathered}
$$

Proof: The proof uses the differentiation rule, already mentioned in Section 3.1:

$$
\frac{d}{d z} z_{2}^{s} F_{1}(s, b, c ; z)=s z^{s-1}{ }_{2} F_{1}(s+1, b, c ; z),
$$

or assuming $s \neq 0$ :

$$
\int z^{s-1}{ }_{2} F_{1}(s+1, b, c ; z) d z=\frac{z^{s}}{s}{ }_{2} F_{1}(s, b, c ; z) .
$$

Set $s=a-1$ with, as fixed before, $a=-\frac{(\delta+n+\pi-\theta)(\beta-\sigma)-\beta(\rho+\pi-n \sigma-\pi \sigma)}{\sigma(\delta+n+\pi-\theta)(1-\beta)}$. We shall see in the next section that the transversality conditions impose $a>1$. Also set $z=$ $\left(1-\frac{\delta+n+\pi-\theta}{\epsilon}\left(\frac{\vartheta_{1}(0)}{\vartheta_{2}(0)}\right)^{-\frac{1-\beta}{\beta}}\right) \exp \left\{-\frac{(1-\beta)(\delta+n+\pi-\theta)}{\beta} t\right\}$, which implies:

$$
d z=-\Theta\left(1-\frac{\delta+n+\pi-\theta}{\epsilon}\left(\frac{\vartheta_{1}(0)}{\vartheta_{2}(0)}\right)^{-\frac{1-\beta}{\beta}}\right) \exp \{-\Theta t\}
$$

where $\Theta=\frac{(1-\beta)(\delta+n+\pi-\theta)}{\beta}$. Putting this together in the integration formula just above, and after some tedious but trivial computations, we get the result stated in the proposition. 


\section{Optimal trajectories}

We now move to characterize the optimal trajectories. Using the transversality conditions, we shall first identify the missing values $\vartheta_{1}(0)$ and $\vartheta_{2}(0)$. Then, we turn to characterize the optimal paths for the remaining variables, education time, consumption and output.

\subsection{Optimal stock and shadow price variables: the general case}

Let us start with the physical capital trajectory which is characterized by the equation (23), Proposition 1 and the transversality condition $\lim _{t \rightarrow \infty} \vartheta_{1} K \exp \{-\rho t\}=0$. Using $(22)$, for any $\vartheta_{1}(0)$ and $\vartheta_{2}(0)$ finite and different from zero, it is easy (though algebraically cumbersome) to identify the right restrictions. Having in mind that we have assumed $\delta+\pi+n-\theta>0$, and provided ${ }_{2} F_{1}(0)$ is constant and tends to ${ }_{2} F_{1}(\infty)={ }_{2} F_{1}(a, b, c ; 0)=1$ when $t$ tends to infinity, one finds that the transversality condition holds if and only if:

$$
\beta(\rho+\pi-n \sigma-\pi \sigma)-(\delta+n+\pi-\theta)(\beta-\sigma)>\sigma(1-\beta)(\delta+n+\pi-\theta)>0
$$

and

$K_{0}\left(\frac{\delta+n+\pi-\theta}{\epsilon}\right)^{-\frac{1}{1-\beta}}\left(\frac{\vartheta_{1}(0)}{\vartheta_{2}(0)}\right)^{\frac{1}{\beta}}+\frac{{ }_{2} F_{1}(0) \beta \sigma N_{0} \vartheta_{2}(0)^{-\frac{1}{\sigma}}\left(\frac{\delta+n+\pi-\theta}{\epsilon}\right)^{-\frac{\beta}{\sigma(1-\beta)}}}{(\delta+n+\pi-\theta)(\beta-\sigma)-\beta(\rho+\pi-n \sigma-\pi \sigma)}=0$.

Let us move now to the optimal human capital path. Here the following transversality condition holds $\lim _{t \rightarrow \infty} \vartheta_{2} h \exp \{-\rho t\}=0$. Using (21), (24) and Proposition 2, for any $\vartheta_{1}(0)$ and $\vartheta_{2}(0)$ finite and different from zero, one can identify the restrictions imposed by the transversality conditions, as for physical capital (using the same asymptotic property for the involved hypergeometric functions):

$$
\rho(\delta-\theta)(1-\sigma)+n
$$

and

$$
h_{0}=\frac{(1-\beta) \epsilon \sigma}{-\beta((\delta-\theta)(1-\sigma)+n-\rho)} K_{0}\left(\frac{\vartheta_{1}(0)}{\vartheta_{2}(0)}\right)^{\frac{1}{\beta}} \frac{{ }_{2} \tilde{F}_{1}(0)}{{ }_{2} F_{1}(0)} .
$$

Putting the previous computations together, one can characterize jointly optimal physical and human capital paths with the following proposition, which will be ultimately used to identify the optimal values for $\vartheta_{1}(0)$ and $\vartheta_{2}(0)$.

Proposition 3 Any particular non-explosive solution to the dynamic system (13)-(16) has to satisfy the initial conditions $K_{0}$ and $h_{0}$, as well as the limiting conditions (9) and (10). These ones impose the constraints:

$$
(\delta+n+\pi-\theta)(\beta-\sigma)-\beta(\rho+\pi-n \sigma-\pi \sigma)<-\sigma(1-\beta)(\delta+n+\pi-\theta)<0,
$$




$$
\begin{gathered}
(\delta-\theta)(1-\sigma)+n-\rho<0 \\
\frac{K_{0}}{{ }_{2} F_{1}(0)}\left(\frac{\vartheta_{1}(0)}{\vartheta_{2}(0)}\right)^{\frac{1}{\beta}}=-\frac{\sigma \beta N_{0} \vartheta_{2}(0)^{-\frac{1}{\sigma}}\left(\frac{\delta+n+\pi-\theta}{\epsilon}\right)^{\frac{\sigma-\beta}{\sigma(1-\beta)}}}{(\delta+n+\pi-\theta)(\beta-\sigma)-\beta(\rho+\pi-n \sigma-\pi \sigma)}, \\
\frac{{ }_{2} F_{1}(0)}{{ }_{2} F_{1}(0)}=\frac{(1-\beta) \epsilon \sigma}{-((\delta-\theta)(1-\sigma)+n-\rho) \beta} \frac{K_{0}}{h_{0}}\left(\frac{\vartheta_{1}(0)}{\vartheta_{2}(0)}\right)^{\frac{1}{\beta}} .
\end{gathered}
$$

Conditions (27) and (28) form a system of two equations with two unknowns, $\vartheta_{1}(0)$ and $\vartheta_{2}(0)$. Their values may be determined in the following way: (28) determines a unique value for the ratio $\frac{\vartheta_{1}(0)}{\vartheta_{2}(0)}$, then $(27)$ determines the value of $\vartheta_{2}(0)$, which after multiplying by the value of the ratio itself gives the value of $\vartheta_{1}(0)$. Therefore, solving the system (27)-(28) is crucial to find out the short term and long run behavior of all the variables of the system. The two next propositions give the closed-form solutions of the physical and human capital stocks assuming the system is solvable with a unique and positive solutions. The positivity and monotonicity of the solution trajectories are specifically studied later in this paper.

Proposition 4 Under the equilibrium conditions, if the system (27)-(28) admits a unique positive solution, then

(i) it does exist a unique path for the physical capital stock $K$, starting from $K_{0}$,

$$
\begin{gathered}
K=K_{0}\left(\frac{\vartheta_{1}(0)}{\vartheta_{2}(0)}\right)^{\frac{1}{\beta}}\left(\frac{\epsilon}{\delta+n+\pi-\theta}\right)^{\frac{1}{1-\beta}} \frac{{ }_{2} F_{1}(t)}{{ }_{2} F_{1}(0)} \\
\cdot \exp \left\{\frac{(\delta+n+\pi-\theta)(\beta-\sigma)-\beta(\rho+\pi-n \sigma)}{\beta \sigma} t\right\} \\
\cdot\left[-1+\exp \left\{\frac{(1-\beta)(\delta+n+\pi-\theta)}{\beta} t\right\}+\frac{\delta+n+\pi-\theta}{\epsilon}\left(\frac{\vartheta_{1}(0)}{\vartheta_{2}(0)}\right)^{-\frac{1-\beta}{\beta}}\right]^{\frac{1}{1-\beta}} ;
\end{gathered}
$$

(ii) this equilibrium path shows transitional dynamics, approaching asymptotically to the unique positive balanced growth path

$$
\bar{K}=\frac{K_{0}}{{ }_{2} F_{1}(0)}\left(\frac{\vartheta_{1}(0)}{\vartheta_{2}(0)}\right)^{\frac{1}{\beta}}\left(\frac{\epsilon}{\delta+n+\pi-\theta}\right)^{\frac{1}{1-\beta}} \exp \left\{\frac{\delta+n-\theta-\rho+n \sigma}{\sigma} t\right\},
$$

along which, given $\delta+n>\theta+\rho, K$ grows permanently at a positive constant rate $\bar{g}_{K}=\frac{\delta+n-\theta-\rho+n \sigma}{\sigma}$.

The proof is obvious and uses the solution expressions found out in the previous section and the transversality conditions corresponding to physical capital accumulation in Proposition 3 just above. The same kind of results can be established for human capital. 
Proposition 5 Under the equilibrium conditions, if the system (27)-(28) admits a unique positive solution, then:

(i) it does exist a unique path for the human capital stock $h$, starting from $h_{0}$,

$$
h=h_{0} \frac{{ }_{2} \tilde{F}_{1}(t)}{\widetilde{F}_{1}(0)} \exp \left\{\frac{\delta+n-\theta-\rho}{\sigma} t\right\}
$$

(ii) this equilibrium path shows transitional dynamics, approaching asymptotically to the unique positive balanced growth path

$$
\bar{h}=\frac{h_{0}}{{ }_{2} \widetilde{F}_{1}(0)} \exp \left\{\frac{\delta+n-\theta-\rho}{\sigma} t\right\},
$$

along which, given $\delta+n>\theta+\rho$, h grows permanently at a positive constant rate $\bar{g}_{h}=$ $\frac{\delta+n-\theta-\rho}{\sigma}$.

We will now concentrate on the system (27)-(28), which is necessary to solve if we want to complete the two previous propositions and to get a more precise characterization of the solution paths in terms of positiveness and monotonicity. We start with the special case $\beta=\sigma$, first identified and studied by Xie (1994), and recently re-explored by Boucekkine and Ruiz-Tamarit (2004). We tackle afterwards the much harder case $\beta \neq \sigma$. Before, the two following lemmas are crucially needed.

Lemma 1 For $z<1$, the ratio $\frac{{ }_{2} F_{1}(0)}{{ }_{2} \widetilde{F}_{1}(0)}=\frac{{ }_{2} F_{1}(b, a, a+1 ; z)}{{ }_{2} F_{1}(b, a-1, a+1 ; z)}$ is an increasing function of $z$ when $b>0$, decreasing when $b<0$ and constant when $b=0$.

Proof: For both Gaussian hypergeometric functions involved in the Lemma, the Euler integral representation applies. So, we have to study the derivative of the ratio of integrals:

$$
\frac{\int_{0}^{1} t^{a-1}(1-t z)^{-b} d t}{\int_{0}^{1} t^{a-2}(1-t)(1-t z)^{-b} d t}
$$

which amounts to signing an expression $\Delta$ given by:

$$
\begin{aligned}
& \frac{\Delta}{b}=\int_{0}^{1} t^{a}(1-t z)^{-b-1} d t \int_{0}^{1} t^{a-2}(1-t)(1-t z)^{-b} d t \\
& -\int_{0}^{1} t^{a-1}(1-t z)^{-b} d t \int_{0}^{1} t^{a-1}(1-t)(1-t z)^{-b-1} d t .
\end{aligned}
$$

The trick now is to rewrite the right hand side as a double integral:

$$
\int_{0}^{1} \int_{0}^{1}\left(x^{a}(1-x z)^{-b-1} y^{a-2}(1-y)(1-y z)^{-b}-x^{a-1}(1-x z)^{-b} y^{a-1}(1-y)(1-y z)^{-b-1}\right) d x d y
$$


which can be factorized as

$$
\int_{0}^{1} \int_{0}^{1} X(x, y, z) x(1-y)(x-y) d x d y
$$

where $X(x, y, z)=x^{a-2}(1-x z)^{-b-1} y^{a-2}(1-y z)^{-b-1}$. Notice that $X(x, y, z)$ is positive, and more importantly, symmetric in $x$ and $y$. Now, it is enough to notice that the double integral above remains the same if we permute the integration variables $x$ and $y$. Hence, summing the original double integral and the same integral with permuted integration variables gives twice the value of the integral. The proof is finished by noticing that the sign of this sum is exactly determined by the sign of $x(1-y)(x-y)+y(1-x)(y-x)$, since $X(x, y, z)$ is positive and symmetric in $(x, y)$. But the expression just above is just equal to $(x-y)^{2}$. Hence, the sign of $\Delta$ is exactly the sign of $b$, and we get the proof of the Lemma.

Lemma 1 establishes the monotonicity properties needed. The next one is needed to establish the uniqueness of the optimal solution paths.

Lemma 2 The $z$-function $\frac{2 F_{1}(b, a, a+1 ; z)}{{ }_{2} F_{1}(b, a-1, a+1 ; z)}-\Omega(1-z)^{-\frac{1}{1-\beta}}$ where

$$
\Omega=\frac{(1-\beta) \epsilon \sigma}{-((\delta-\theta)(1-\sigma)+n-\rho) \beta} \frac{K_{0}}{h_{0}}\left(\frac{\delta+n+\pi-\theta}{\epsilon}\right)^{\frac{1}{1-\beta}},
$$

is strictly decreasing when $b<0$. When $b>0$, the function is first increasing, then it becomes strictly decreasing (hump-shaped).

The proof is reported in the appendix.

\subsection{The case $\beta=\sigma$}

This is the easiest case to solve. Indeed when $\beta=\sigma$, then both ${ }_{2} F_{1}(0)$ and ${ }_{2} \tilde{F}_{1}(0)$, which appear in the system (27)-(28), will degenerate into positive constants, independent of the ratio $\frac{\vartheta_{1}(0)}{\vartheta_{2}(0)}$. This is a direct consequence of Lemma 1 , but this property can be derived even more trivially. Indeed, as mentioned in Section 3.1, the gaussian hypergeometric functions ${ }_{2} F_{1}(a, b, c ; z)$ degenerate into polynomials of degree $n$ in $\mathrm{z}$ if $a$ or $b$ are equal to $-n$, where $n=0,1,2, \ldots$ If $\beta=\sigma$, then $b=0$ and therefore, the involved hypergeometric functions are polynomials of degree 0 of $z$, which turns out to be equal, by Proposition 1 and Proposition 2, to $A_{0}$, defined earlier as:

$$
A_{0}=\frac{\delta+n+\pi-\theta}{\epsilon}\left(\frac{\vartheta_{1}(0)}{\vartheta_{2}(0)}\right)^{-\frac{1-\beta}{\beta}}-1 .
$$


Thus, the hypergeometric terms are constant, independent of the ratio $\frac{\vartheta_{1}(0)}{\vartheta_{2}(0)}$. The constant should be positive because by condition (25) in Proposition 3, $a>1$. Since $c=a+1$, the Pochhammer terms appearing in the hypergeometric are all positive, and the terms ${ }_{2} F_{1}(0)$ and ${ }_{2} \widetilde{F}_{1}(0)$ are necessarily positive. The system $(27)-(28)$ has then trivially a unique positive solution. Using Proposition 4 and Proposition 5, it is then quite trivial to draw the following conclusions.

Proposition 6 Under the equilibrium conditions, when $\sigma=\beta$ the system (27)-(28) has a unique positive solution for $\vartheta_{1}(0)$ and $\vartheta_{2}(0)$. Moreover, the optimal path of physical capital stock given in (29) is positive and shows up transitional dynamics to the balanced growth path given by (30). On the other hand, the optimal path of human capital stock given in (31) is positive, strictly monotonic, but does not show up any transitional dynamics to the balanced growth path given by (32)

The proof is trivial when putting $\beta=\sigma$ in (29) and (31), and after noticing that ${ }_{2} F_{1}(t)$ and ${ }_{2} \tilde{F}_{1}(t)$, just like ${ }_{2} F_{1}(0)$ and ${ }_{2} \widetilde{F}_{1}(0)$, degenerate into strictly positive constants under $\beta=\sigma$. The results are completely in line with those of Boucekkine and Ruiz-Tamarit (2004), who use a completely different method to solve the Lucas model in the same parametric case. In particular, adjustment to the long run equilibrium relies on physical capital, in the absence of any transition dynamics for human capital. Let us now move to the case $\beta \neq \sigma$.

\subsection{The case $\beta \neq \sigma$}

We are in the case where $b \neq 0$. As mentioned before, finding a solution to the system (27)-(28) amounts to solving the equation in the ratio $\frac{\vartheta_{1}(0)}{\vartheta_{2}(0)}$ :

$$
\frac{{ }_{2} F_{1}(0)}{{ }_{2} F_{1}(0)}=\frac{(1-\beta) \epsilon \sigma}{-((\delta-\theta)(1-\sigma)+n-\rho) \beta} \frac{K_{0}}{h_{0}}\left(\frac{\vartheta_{1}(0)}{\vartheta_{2}(0)}\right)^{\frac{1}{\beta}} \text {. }
$$

It is readily shown that this equation is equivalent to an equation in $z=-A_{0}=1-$ $\frac{\delta+n+\pi-\theta}{\epsilon}\left(\frac{\vartheta_{1}(0)}{\vartheta_{2}(0)}\right)^{-\frac{1-\beta}{\beta}}$, given by:

$$
\frac{{ }_{2} F_{1}(b, a, a+1 ; z)}{{ }_{2} F_{1}(b, a-1, a+1 ; z)}=\Omega(1-z)^{-\frac{1}{1-\beta}}
$$

where $\Omega$ is already defined in the statement of Lemma 2. Notice that under condition (26), $\Omega>0$. Using the balanced path equations for physical and human capital, (30) and (32) respectively, and the condition (28) arising from the transversality conditions, one can show immediately that: (i) $\Omega<1$ is equivalent to $\frac{K_{0}}{h_{0}}<\frac{\bar{K}}{N h}$; (ii) $\Omega=1$ is equivalent 
to $\frac{K_{0}}{h_{0}}=\frac{\bar{K}}{N h}$, and (iii) $\Omega>1$ is equivalent to $\frac{K_{0}}{h_{0}}>\frac{\bar{K}}{N h}$. The situation (i) features a case for radial convergence from below, while (iii) is a case for convergence from above. Case (ii) is a situation where initially the economy is already, say, on the good radius. In this context, we are close to the concepts put forward by Caballé and Santos (1993). However, thanks to our closed-form solutions for all the variables in level, we are able to go beyond radial convergence and prove convergence in level in the Lucas-Uzawa model. Let us study the three situations listed above. The following lemma will be useful along the way.

Lemma 3 The ratio $\frac{{ }_{2} F_{1}(0)}{{ }_{2} F_{1}(0)}=\frac{{ }_{2} F_{1}(b, a, a+1 ; z)}{{ }_{2} F_{1}(b, a-1, a+1 ; z)}$ is strictly below (Resp. above) function $\kappa_{0}(z)=\Omega(1-z)^{-\frac{1}{1-\beta}}$ when $z$ is close enough to 1 (Resp. tends to $\left.-\infty\right)$.

Proof: The proof is quite easy. The position of the ratio $\frac{{ }_{2} F_{1}(b, a, a+1 ; z)}{2 F_{1}(b, a-1, a+1 ; z)}$ with respect to $\kappa_{0}(z)$ is determined by the sign of the difference

$$
a \int_{0}^{1} t^{a-1}(1-z t)^{-b} d t-\Omega(a-1)(1-z)^{-\frac{1}{1-\beta}} \int_{0}^{1} t^{a-2}(1-t)(1-z t)^{-b} d t
$$

which can be written as:

$$
\int_{0}^{1} t^{a-2}(1-z t)^{-b} \kappa_{1}(z, t) d t
$$

where $\kappa_{1}(z, t)=a t-\Omega(a-1)(1-z)^{-\frac{1}{1-\beta}}(1-t)$. Now, it's enough to notice that the latter function is an affine function in $t$, positive when $t>t_{1}$, negative when $t<t_{1}$ and nil when $t=t_{1}$, with

$$
t_{1}=\frac{\Omega(a-1)(1-z)^{-\frac{1}{1-\beta}}}{a+\Omega(a-1)(1-z)^{-\frac{1}{1-\beta}}} .
$$

When $z$ tends to $1, t_{1}$ tends to 1 , and since the integration variable $t$ is comprised between 0 and 1 , this means that $\kappa_{1}(z, t)$ tends to a negative function on the whole integration interval when $z$ tends to 1 . Henceforth, the ratio of functions $\frac{{ }_{2} F_{1}(b, a, a+1 ; z)}{{ }_{2} F_{1}(b, a-1, a+1 ; z)}$ does fall below function $\kappa_{0}(z)$ in this neighborhood.

When $z$ tends to $-\infty, t_{1}$ goes to zero, and we get the opposite result.

We now study the three situations outlined before Lemma 3. In situation (i): $\Omega<1$. Notice that $z=0, \frac{{ }_{2} F_{1}(b, a, a+1 ; 0)}{2 F_{1}(b, a-1, a+1 ; 0)}=1>\Omega=\kappa_{0}(0)$. By Lemma 3, we know, that $\frac{{ }_{2} F_{1}(b, a, a+1 ; z)}{{ }_{2} F_{1}(b, a-1, a+1 ; z)}<\kappa_{0}(z)$ when $z$ tends to 1 . Therefore, by continuity, $\frac{{ }_{2} F_{1}(b, a, a+1 ; z)}{{ }_{2} F_{1}(b, a-1, a+1 ; z)}=\kappa_{0}(z)$ for some $z \in\left(\begin{array}{ll}0 & 1\end{array}\right)$, and equation (33) has at least one solution in this interval. Lemma 1 and 2 ensure uniqueness. 
In situation (iii), $\Omega>1$, so $\frac{2 F_{1}(b, a, a+1 ; 0)}{{ }_{2} F_{1}(b, a-1, a+1 ; 0)}=1<\Omega=\kappa_{0}(0)$. But, by Lemma 3, $\frac{{ }_{2} F_{1}(b, a, a+1 ; z)}{{ }_{2} F_{1}(b, a-1, a+1 ; z)}>\kappa_{0}(z)$ when $z$ tends to $-\infty$. Therefore, equation (33) has at least one solution, and this solution is strictly negative. Again, Lemma 1 and 2 ensure uniqueness. In situation (ii), $\Omega=1$, which immediately implies that $z=0$ is the unique solution to equation (33). It follows that the system (27) and (28) has a unique solution, which ultimately means the transversality conditions of the considered optima control problem do yield a unique admissible couple $\left(\vartheta_{1}(0), \vartheta_{2}(0)\right)$. Henceforth, the optimal trajectories given in Proposition 4 and 5 are uniquely defined. This is the crucial step of our treatment, the next build on the properties established along the way to achieve this step. The remaining characterization work (of the optimal trajectories) is briefly reported in the two next sections.

\subsection{On the monotonicity of the optimal paths}

An important outcome of our approach is the explicit study of the monotonicity of the optimal trajectories of the variables in level, an exploration typically unallowed in the alternative approaches. The next proposition states that monotonicity may not be a general property of optimal physical and human capital stocks' dynamics.

Proposition 7 Under the equilibrium conditions, if $\delta+n>\theta+\rho$, then the physical and human capital stocks are positive along the transition to the balanced growth paths. The transitions are not necessarily monotonic.

Using the characterization (29) of physical capital dynamics, one is able to express these dynamics

$$
K=K_{0}\left(\frac{\vartheta_{1}(0)}{\vartheta_{2}(0)}\right)^{\frac{1}{\beta}}\left(\frac{\epsilon}{\delta+n+\pi-\theta}\right)^{\frac{1}{1-\beta}} \frac{{ }_{2} F_{1}(t)}{{ }_{2} F_{1}(0)} \exp \left(\frac{\delta+n-\theta-\rho+n \sigma}{\sigma} t\right) \beta_{0}(t),
$$

where

${ }_{2} F_{1}(t)={ }_{2} F_{1}\left(a, b ; c ;\left(1-\frac{\delta+n+\pi-\theta}{\epsilon}\left(\frac{\vartheta_{1}(0)}{\vartheta_{2}(0)}\right)^{-\frac{1-\beta}{\beta}}\right) \exp \left\{-\frac{(1-\beta)(\delta+n+\pi-\theta)}{\beta} t\right\}\right)$,

and $\beta_{0}(t)=\left(1-z^{o} \exp \left(-\frac{1-\beta}{\beta}(\delta+n+\pi-\theta) t\right)\right)^{\frac{1}{1-\beta}}, z^{o}$ being the unique solution to (33) as discussed in Section 4.3. The positivity is trivial since the unique possibly negative term is $\beta_{0}(t)$. However, this term is always positive. When $\Omega<1$ (convergence from below), $0<z^{o}<1$ and $\beta_{0}(t)$ is strictly increasing from $\beta_{0}(0)=\left(1-z^{o}\right)^{\frac{1}{1-\beta}}>0$. When 
$\Omega>1$ (convergence from above), $z^{o}<0$ and $\beta_{0}(t)$ is strictly decreasing from $\beta_{0}(0)=$ $\left(1-z^{o}\right)^{\frac{1}{1-\beta}}>0$ to 1 . Hence, positivity is always ensured.

More importantly, monotonicity is by no way guaranteed for all values of $b$ and whatever the position of $\Omega$ with respect to 1 . For example, suppose $\Omega<1$, which means $0<z^{o}<1$. Let us have a look at the expression of $K(t)$ just above. It's the product of three time dependent functions. The first one, ${ }_{2} F_{1}(t)$, is strictly increasing when $b<0$ and strictly decreasing when $b>0$. The second and third ones are increasing. It follows that while $K(t)$ is unambiguously increasing when $b<0$, it may be non-monotonic if $b>0$. Things are even more ambiguous when $\Omega>1$, implying a negative $z^{o}$ value. In such a case, the third term becomes decreasing, and there is no unambiguous parametric case at all in such a situation. Section 5 will present some parametric cases in which the optimal capital stock is actually non-monotonic, but it is already clear that there is no analytical basis for monotonicity of the transition dynamics, at least for the physical capital stock.

The same observation has to be made even for the apparently simpler human capital dynamics, given by equation (31):

$$
h(t)=h_{0} \frac{{ }_{2} \widetilde{F}_{1}(t)}{{ }_{2} F_{1}(0)} \exp \left\{\frac{\delta+n-\theta-\rho}{\sigma} t\right\} .
$$

When $\Omega<1$ and $b<0, h(t)$ is necessarily strictly increasing. When $\Omega>1$ and $b>0$, $h(t)$ is also unambiguously strictly increasing. Non-monotonicity is a priori possible in the remaining cases, we shall not go much further regarding this cumbersome algebraic issue here. ${ }^{6}$

A final observation concerns the behavior of the ratio $\frac{K}{N h}$, which is central in the analysis of Caballé and Santos for example. In our numerical experiments reported partly in Section 5, this ratio shows up a monotonic behavior while $K(t)$ generally does not, as we mentioned above. Proposition 8 just below proves analytically this result in the case $b=0$.

Proposition 8 Under the equilibrium conditions, if $\delta+n>\theta+\rho$ and $b=0$, the ratio $\frac{K}{N h}$ follows a monotonic optimal path, while the physical capital stock is strictly increasing when $\Omega<1$ but is non-monotonic for a non-zero measure set of values of the ratio $\frac{K_{0}}{h_{0}}$ when $\Omega>1$ provided $\frac{\beta^{-\frac{\beta}{1-\beta}}}{1-\beta}<\frac{\delta+n+\pi-\theta}{\delta+n-\theta-\rho+n \sigma}$.

\footnotetext{
${ }^{6}$ To fully identify the non-monotonicity conditions, one has to differentiate the considered variables, say $x(t)$, and study the conditions under which $x^{\prime}(0)<0$. Since Section 5 include some transparent illustrations of non-monotonicity for $K(t), h(t), c(t)$ and $Y(t)$, it's enough to make the point.
} 
The proof is in the appendix. This property highlights another potential problem deriving from the dimension reduction strategy: Ratios of variables may "hide" the nonmonotonicities and eventually rich dynamic contents of the variables. Therefore if one is interested in explaining the role of physical vs human capital dynamics in the business cycles, this strategy may be misleading.

\subsection{Completing the job: optimal production time, consumption and output dynamics}

We now give a complete characterization of optimal paths for variables production time, consumption per capita, output and the ratio physical to human capital. In particular, the next proposition proves that the dynamics of production time should be monotonic.

Proposition 9 Under the equilibrium conditions, if $\delta+n>\theta+\rho$ then:

(i) it does exist a unique, interior and monotonous path for the fraction of non-leisure time devoted to goods production

$$
0<u=-\frac{(\delta-\theta)(1-\sigma)+n-\rho_{2} F_{1}(t)}{\sigma \delta} \frac{{ }_{2} \widetilde{F}_{1}(t)}{\sigma \delta} ;
$$

(ii) this equilibrium path, which starts from $u(0)=-\frac{(\delta-\theta)(1-\sigma)+n-\rho}{\sigma \delta} \frac{{ }_{2} F_{1}(0)}{\widetilde{F}_{1}(0)}$, shows transitional dynamics converging asymptotically to the interior constant value

$$
0<\bar{u}=-\frac{(\delta-\theta)(1-\sigma)+n-\rho}{\sigma \delta}<1 ;
$$

(iii) the initial fraction of non-leisure time devoted to goods production satisfies the constraint $0<u(0)<1$ if and only if $\frac{K_{0}}{h_{0}}\left(\frac{\vartheta_{1}(0)}{\vartheta_{2}(0)}\right)^{\frac{1}{\beta}}<\frac{\delta \beta}{(1-\beta) \epsilon}$.

Proof. From (12), given (21), (22), (29) and (31) we get (34). Then, taking the limit as $t$ tends to infinity we obtain (35), where $\bar{u}$ is strictly interior for $\delta+n>\theta+\rho$ because of (26) and $\sigma \theta \geq 0$. Monotonicity derives immediately from Lemma 1 . The interiority of $u(0)$ comes from (26), which ensures the lower bound, and the iff condition from (iii), which under (28) guarantees the upper bound. Finally, given that $u$ follows a monotonous convergent trajectory, the interiority of this one is a direct consequence of the interiority of both $u(0)$ and $\bar{u}$.

The dynamics of optimal consumption per capita are quite immediately derived from the dynamics of the shadow price $\vartheta_{1}(t),(22)$, given the optimality condition (11). 
Proposition 10 Under the equilibrium conditions:

(i) it does exist a unique and positive path for consumption per capita $c$, starting from $c(0)=\left(\vartheta_{1}(0)\right)^{-\frac{1}{\sigma}}$,

$$
\begin{gathered}
c=\left(\frac{\epsilon}{\delta+n+\pi-\theta}\right)^{\frac{\beta}{\sigma(1-\beta)}}\left(\vartheta_{2}(0)\right)^{-\frac{1}{\sigma}} \exp \left\{-\frac{\rho+\pi}{\sigma} t\right\} \\
\cdot\left[-1+\exp \left\{\frac{(1-\beta)(\delta+n+\pi-\theta)}{\beta} t\right\}+\frac{\delta+n+\pi-\theta}{\epsilon}\left(\frac{\vartheta_{1}(0)}{\vartheta_{2}(0)}\right)^{-\frac{1-\beta}{\beta}}\right]^{\frac{\beta}{\sigma(1-\beta)}}
\end{gathered}
$$

(ii) this optimal path shows transitional dynamics, approaching asymptotically to the unique positive balanced growth path

$$
\bar{c}=\left(\vartheta_{2}(0)\right)^{-\frac{1}{\sigma}}\left(\frac{\epsilon}{\delta+n+\pi-\theta}\right)^{\frac{\beta}{\sigma(1-\beta)}} \exp \left\{\frac{\delta+n-\theta-\rho}{\sigma} t\right\},
$$

along which, given $\delta+n>\theta+\rho$, c grows permanently at a positive constant rate $\bar{g}_{c}=$ $\frac{\delta+n-\theta-\rho}{\sigma}$.

Indeed if we combine (11) and (22), we get (36), and taking the limit as $t$ tends to infinity we obtain (37). From Propositions 4, 5, 7 and 9, we can now directly deduce the following corollaries, which focus on the dynamics of the aggregate production level, the relative shadow prices and the ratio between capital stocks.

Corollary 1 Under the equilibrium conditions, the following properties hold:

(i) it does exist a unique and positive path for production $Y$, starting from $Y(0)=$ $A K_{0}\left(\frac{\vartheta_{1}(0)}{\vartheta_{2}(0)}\right)^{\frac{1-\beta}{\beta}}\left(\frac{(1-\beta) \epsilon N_{0}}{\delta \beta}\right)^{1-\beta}=A^{\frac{1}{\beta}} K_{0}\left(\frac{\vartheta_{1}(0)}{\vartheta_{2}(0)}\right)^{\frac{1-\beta}{\beta}}\left(\frac{(1-\beta) N_{0}}{\delta}\right)^{\frac{1-\beta}{\beta}}$,

$$
\begin{gathered}
Y=Y(0) \frac{\vartheta_{1}(0)}{\vartheta_{2}(0)}\left(\frac{\epsilon}{\delta+n+\pi-\theta}\right)^{\frac{\beta}{1-\beta}} \frac{{ }_{2} F_{1}(t)}{{ }_{2} F_{1}(0)} \exp \left\{\frac{\delta+n-\theta-\rho-\sigma(\delta+\pi-\theta)}{\sigma} t\right\} \\
\cdot\left[-1+\exp \left\{\frac{(1-\beta)(\delta+n+\pi-\theta)}{\beta} t\right\}+\frac{\delta+n+\pi-\theta}{\epsilon}\left(\frac{\vartheta_{1}(0)}{\vartheta_{2}(0)}\right)^{-\frac{1-\beta}{\beta}}\right]^{\frac{\beta}{1-\beta}} ;
\end{gathered}
$$

(ii) this equilibrium path shows transitional dynamics, approaching asymptotically to the unique positive balanced growth path

$$
\bar{Y}=\frac{Y(0)}{{ }_{2} F_{1}(0)} \frac{\vartheta_{1}(0)}{\vartheta_{2}(0)}\left(\frac{\epsilon}{\delta+n+\pi-\theta}\right)^{\frac{\beta}{1-\beta}} \exp \left\{\frac{\delta+n-\theta-\rho+n \sigma}{\sigma} t\right\}
$$

along which, given $\delta+n>\theta+\rho, Y$ grows permanently at a positive constant rate $\bar{g}_{Y}=$ $\frac{\delta+n-\theta-\rho+n \sigma}{\sigma}$. 
Given that $Y=A K^{\beta} u^{1-\beta} h^{1-\beta}$, Propositions 4, 5, 7 and 9 and some additional algebra suffice to prove this corollary. We end our characterization by a brief statement on the relative price $\frac{\vartheta_{1}}{\vartheta_{2}}$ and the ratio $\frac{K}{h}$.

Corollary 2 Under the equilibrium conditions, we have:

(i) it does exist a unique and positive path for the relative prices $\frac{\vartheta_{1}}{\vartheta_{2}}$ and a unique, positive path for the ratio $\frac{K}{h}$, starting from $\frac{\vartheta_{1}(0)}{\vartheta_{2}(0)}$ and $\frac{K_{0}}{h_{0}}$ respectively,

$$
\begin{gathered}
\frac{\vartheta_{1}}{\vartheta_{2}}=\left(\frac{\delta+n+\pi-\theta}{\epsilon}\right)^{\frac{\beta}{1-\beta}} \exp \{(\delta+\pi-\theta) t\} \\
\cdot\left[-1+\exp \left\{\frac{(1-\beta)(\delta+n+\pi-\theta)}{\beta} t\right\}+\frac{\delta+n+\pi-\theta}{\epsilon}\left(\frac{\vartheta_{1}(0)}{\vartheta_{2}(0)}\right)^{-\frac{1-\beta}{\beta}}\right]^{-\frac{\beta}{1-\beta}}, \quad(40) \\
\frac{K}{h}=\frac{K_{0}}{h_{0}}\left(\frac{\vartheta_{1}(0)}{\vartheta_{2}(0)}\right)^{\frac{1}{\beta}}\left(\frac{\epsilon}{\delta+n+\pi-\theta}\right)^{\frac{1}{1-\beta}} \frac{\tilde{F}_{2}(0)}{{ }_{2} F_{1}(0)} \frac{{ }_{2} F_{1}(t)}{\tilde{F}_{1}(t)} \exp \left\{-\frac{\delta+n+\pi-\theta-\beta n}{\beta} t\right\} \\
\cdot\left[-1+\exp \left\{\frac{(1-\beta)(\delta+n+\pi-\theta)}{\beta} t\right\}+\frac{\delta+n+\pi-\theta}{\epsilon}\left(\frac{\vartheta_{1}(0)}{\vartheta_{2}(0)}\right)^{-\frac{1-\beta}{\beta}}\right]^{\frac{1}{1-\beta}} ;
\end{gathered}
$$

(ii) these equilibrium paths move asymptotically showing transitional dynamics, and converge respectively to the unique and positive balanced growth paths

$$
\begin{gathered}
\left(\frac{\overline{\vartheta_{1}}}{\vartheta_{2}}\right)=\left(\frac{\delta+n+\pi-\theta}{\epsilon}\right)^{\frac{\beta}{1-\beta}} \exp \{-n t\} \\
\left(\frac{\bar{K}}{h}\right)=-\frac{((\delta-\theta)(1-\sigma)+n-\rho) \beta}{(1-\beta) \epsilon \sigma}\left(\frac{\epsilon}{\delta+n+\pi-\theta}\right)^{\frac{1}{1-\beta}} \exp \{n t\} .
\end{gathered}
$$

Again the proof is trivial: taking (21) and (22) on the one hand, (29) and (31) on the other, and dividing between them we get (40) and (41), respectively. Moreover, taking respectively the limit as $t$ tends to infinity we derive (42) and, using (28), also (43). Using the value of $\epsilon$ and the population growth process, the two above equations may be rewritten as

$$
\begin{gathered}
N\left(\frac{\overline{\vartheta_{1}}}{\vartheta_{2}}\right)=\frac{\delta}{(1-\beta) A}\left(\frac{\delta+n+\pi-\theta}{\beta A}\right)^{\frac{\beta}{1-\beta}}>0 \\
\frac{1}{N}\left(\frac{\bar{K}}{h}\right)=-\frac{(\delta-\theta)(1-\sigma)+n-\rho}{\sigma \delta}\left(\frac{\beta A}{\delta+n+\pi-\theta}\right)^{\frac{1}{1-\beta}}>0 .
\end{gathered}
$$


All these results are general in the sense that they encompass the three different subcases arising from the relationship between the parameters representing the inverse of the intertemporal elasticity of substitution, $\sigma$, and the physical capital share, $\beta$. These subcases have drawn great attention in growth literature because they cause different patterns of dynamic behavior. However, what we supply here is a compact general solution for all of them, based on the hypergeometric function with $a>1, \tilde{a}>0$ and $c>2$ because of the parameter constraints (25) and (26) implied by transversality conditions, and with $b \gtreqless 0$ depending on $\sigma \gtreqless \beta$.

\section{Economic discussion of the results}

\subsection{The effect of changes in structural parameters on the bal- anced growth paths}

In this subsection we analyze how changes in structural parameters will affect: $i)$ the common long-run rate of growth, $\bar{g}=\frac{\delta+n-\theta-\rho}{\sigma}$, corresponding to physical and human capital stock, physical output and consumption, when all of them are measured in per capita terms; ii) the long-run fraction of non-leisure time devoted to goods production, $\bar{u}=$ $-\frac{(\delta-\theta)(1-\sigma)+n-\rho}{\sigma \delta}$; iii) the long-run ratio between capital stocks, $\left(\frac{\bar{K}}{N h}\right)=-\frac{(\delta-\theta)(1-\sigma)+n-\rho}{\sigma \delta}\left(\frac{\beta A}{\delta+n+\pi-\theta}\right)^{\frac{1}{1-\beta}}$;

iv) the long-run relationship between output and physical capital, $\left(\frac{\bar{Y}}{K}\right)=\frac{\delta+n+\pi-\theta}{\beta}$; and $\left.v\right)$ the long-run ratio consumption to physical capital, $\left(\frac{\bar{N} c}{K}\right)=\frac{\delta+n+\pi-\theta}{\beta}-\frac{\delta+n-\theta-\rho}{\sigma}-n-\pi$. An increase (decrease) in the productivity of educational sector, $\delta$, increases (decreases) the balanced growth rate. The productivity of goods sector, $A$, does not affect the balanced growth rate. An increase (decrease) in the rate of population growth, $n$, increases (decreases) the balanced growth rate. An increase (decrease) in the elasticity of intertemporal substitution, $\sigma^{-1}$, increases (decreases) the balanced growth rate. A decrease (increase) in the human capital depreciation rate, $\theta$, increases (decreases) the balanced growth rate. The depreciation rate of physical capital, $\pi$, does not affect the balanced growth rate. A decrease (increase) in the time preference rate, $\rho$, increases (decreases) the balanced growth rate. The elasticity of output with respect to physical capital, $\beta$, does not affect the balanced growth rate.

In the normal case with $\sigma>\beta$, if $\sigma \frac{\rho-n+\theta}{\theta}\left(\sigma<\frac{\rho-n+\theta}{\theta}\right)$ an increase (decrease) in the productivity of educational sector, $\delta$, increases the long-run fraction of non-leisure time devoted to goods production. In the exogenous growth and paradoxical cases, with $\sigma=\beta$ and $\sigma<\beta$ respectively, a decrease (increase) in the productivity of educational sector, 
$\delta$, increases (decreases) the long-run fraction of non-leisure time devoted to goods production. The productivity of goods sector, $A$, does not affect this fraction in any of the previous cases. A decrease (increase) in the rate of population growth, $n$, increases (decreases) the long-run fraction of non-leisure time devoted to goods production.

In all of the cases a decrease (increase) in the elasticity of intertemporal substitution, $\sigma^{-1}$, increases (decreases) the long-run fraction of non-leisure time devoted to goods production. In the normal case, if $\sigma>1(\sigma<1)$ a decrease (increase) in the human capital depreciation rate, $\theta$, increases the long-run fraction of non-leisure time devoted to goods production. In the exogenous growth and paradoxical cases an increase (decrease) in the human capital depreciation rate, $\theta$, increases (decreases) the long-run fraction of non-leisure time devoted to goods production. Instead, the depreciation rate of physical capital, $\pi$, does not affect the long-run fraction of non-leisure time devoted to goods production. An increase (decrease) in the time preference rate, $\rho$, increases (decreases) the long-run fraction of non-leisure time devoted to goods production. The elasticity of output with respect to physical capital, $\beta$, does not affect the long-run fraction of non-leisure time devoted to goods production.

In the normal case with $\sigma>\beta$, if $\sigma>\frac{\rho-n+\theta}{\theta}$ the effect of the productivity of educational sector, $\delta$, on the long-run physical-to-human-capital ratio is ambiguous, but if $\sigma<\frac{\rho-n+\theta}{\theta}$, as well as in the exogenous growth and paradoxical cases with $\sigma=\beta$ and $\sigma<\beta$ respectively, a decrease (increase) in the productivity of educational sector, $\delta$, increases (decreases) the long-run physical-to-human-capital ratio. In any of the previous cases, an increase (decrease) in the productivity of goods sector, $A$, increases (decreases) the long-run physical-to-human-capital ratio. A decrease (increase) in the rate of population growth, $n$, increases (decreases) the long-run physical-to-human-capital ratio. A decrease (increase) in the elasticity of intertemporal substitution, $\sigma^{-1}$, increases (decreases) the long-run physical-to-human-capital ratio. In the normal case with $\sigma>\beta$, if $\sigma>1$ the effect of the human capital depreciation rate, $\theta$, on the long-run physical-to-human-capital ratio is ambiguous, but if $\sigma<1$, as well as in the exogenous growth and paradoxical cases with $\sigma=\beta$ and $\sigma<\beta$ respectively, an increase (decrease) in the human capital depreciation rate, $\theta$, increases (decreases) the long-run physical-to-human-capital ratio. In any of the previous cases, a decrease (increase) in the depreciation rate of physical capital, $\pi$, increases (decreases) the long-run physical-to-human-capital ratio. An increase (decrease) in the time preference rate, $\rho$, increases (decreases) the long-run physical-tohuman-capital ratio. An increase (decrease) in the elasticity of output with respect to 
physical capital, $\beta$, increases (decreases) the long-run physical-to-human-capital ratio.

An increase (decrease) in the productivity of educational sector, $\delta$, increases (decreases) the long-run output to physical capital ratio. The productivity of goods sector, $A$, does not affect this ratio. An increase (decrease) in the rate of population growth, $n$, increases (decreases) the long-run output to physical capital ratio. The elasticity of intertemporal substitution, $\sigma^{-1}$, does not affect this ratio. A decrease (increase) in the human capital depreciation rate, $\theta$, increases (decreases) the long-run output to physical capital ratio. An increase (decrease) in the depreciation rate of physical capital, $\pi$, increases (decreases) the long-run output to physical capital ratio. The time preference rate, $\rho$, does not affect this ratio. A decrease (increase) in the elasticity of output with respect to physical capital, $\beta$, increases (decreases) the long-run output to physical capital ratio.

In the normal case with $\sigma>\beta$, an increase (decrease) in the productivity of educational sector, $\delta$, increases (decreases) the long-run consumption to physical capital ratio. In the exogenous growth case with $\sigma=\beta$, it does not affect this ratio. In the paradoxical case with $\sigma<\beta$, a decrease (increase) in the productivity of educational sector, $\delta$, increases (decreases) the long-run consumption to physical capital ratio. The productivity of goods sector, $A$, does not affect this ratio in any of the previous cases. In the normal case, if $\sigma>\frac{\beta}{1-\beta}\left(\sigma<\frac{\beta}{1-\beta}\right)$ an increase (decrease) in the rate of population growth, $n$, increases the long-run consumption to physical capital ratio. In the exogenous growth and paradoxical cases a decrease (increase) in the rate of population growth, $n$, increases (decreases) the long-run consumption to physical capital ratio.

In all of the cases a decrease (increase) in the elasticity of intertemporal substitution, $\sigma^{-1}$, increases (decreases) the long-run consumption to physical capital ratio. In the normal case a decrease (increase) in the human capital depreciation rate, $\theta$, increases (decreases) the long-run consumption to physical capital ratio. In the exogenous growth case it does not affect the ratio. In the paradoxical case an increase (decrease) in the human capital depreciation rate, $\theta$, increases (decreases) the long-run consumption to physical capital ratio. Instead, for any of the three cases, an increase (decrease) in the depreciation rate of physical capital, $\pi$, increases (decreases) the long-run consumption to physical capital ratio. An increase (decrease) in the time preference rate, $\rho$, increases (decreases) the long-run consumption to physical capital ratio. A decrease (increase) in the elasticity of output with respect to physical capital, $\beta$, increases (decreases) the long-run consumption to physical capital ratio. 


\subsection{Numerical experiments}

It is well-known that according to the relationship between $\sigma$ and $\beta$ we can identify three complementary cases determining different transitional dynamics for the variables of the model. First, the normal case, which corresponds to $\sigma_{1}>\beta$ and, hence, to $b_{1}>0$. Second, the exogenous growth case, which corresponds to $\sigma_{2}=\beta$ and, hence, to $b_{2}=0$. Finally, the paradoxical case associated with $\sigma_{3}<\beta$ and $b_{3}<0$. In this section we consider the following benchmark economy: $N_{0}=1, n=0, A=1, \delta=0.06, \pi=0.05$, $\theta=0.01, \rho=0.04, \beta=0.45, \sigma_{1}=1.2, \sigma_{2}=0.45, \sigma_{3}=0.3$ and, hence, $\epsilon=6.7491$, the common long-run rates of growth $\bar{g}_{1}=0.008, \bar{g}_{2}=0.022, \bar{g}_{3}=0.03$, the long-run fractions of non-leisure time devoted to goods production $\bar{u}_{1}=0.694, \bar{u}_{2}=0.463, \bar{u}_{3}=0.278$, and the long-run ratios between capitals $\left(\frac{\bar{K}}{N h}\right)_{1}=10.698,\left(\frac{\bar{K}}{N h}\right)_{2}=7.132,\left(\frac{\bar{K}}{N h}\right)_{3}=4.279$.

These parameters lead to the following ones, which contribute to specify the different hypergeometric functions used in this numerical exercise, $a_{1}=1.3409, \tilde{a}_{1}=0.3409, b_{1}=$ $1.1364, c_{1}=2.3409, a_{2}=1.2273, \tilde{a}_{2}=0.2273, b_{2}=0.0, c_{2}=2.2273, a_{3}=1.1364$, $\tilde{a}_{3}=0.1364, b_{3}=-0.9091, c_{3}=2.1364$. On the other hand, the initial values of $K$ and $h$ have been chosen, alternatively, as $K_{0}=1.0$ and $h_{0}=0.25$, and $K_{0}=15$ and $h_{0}=1.0$, to analyze both cases: i) $\left(\frac{K}{N h}\right)_{0}=4<\left(\frac{\bar{K}}{N h}\right)_{i=1,2,3}$ with $u(0)_{1}=0.976, u(0)_{2}=0.463$, $u(0)_{3}=0.274$; and ii) $\left(\frac{K}{N h}\right)_{0}=15>\left(\frac{\bar{K}}{N h}\right)_{i=1,2,3}$ with $u(0)_{1}=0.613, u(0)_{2}=0.463$, $u(0)_{3}=0.359$. That is, convergence from below as well as convergence from above. Under the previous set of parameter values and using (28) we get the following two sets of initial values for the ratio between the shadow prices of physical and human capital: i) $\left(\frac{\vartheta_{1}(0)}{\vartheta_{2}(0)}\right)_{1}=0.0578,\left(\frac{\vartheta_{1}(0)}{\vartheta_{2}(0)}\right)_{2}=0.0413,\left(\frac{\vartheta_{1}(0)}{\vartheta_{2}(0)}\right)_{3}=0.0327$ and ii) $\left(\frac{\vartheta_{1}(0)}{\vartheta_{2}(0)}\right)_{1}=0.0259$, $\left(\frac{\vartheta_{1}(0)}{\vartheta_{2}(0)}\right)_{2}=0.0228,\left(\frac{\vartheta_{1}(0)}{\vartheta_{2}(0)}\right)_{3}=0.0203$.

\subsection{Transitional dynamics}

In this subsection we study how the main variables of the model evolve along their transition to the balanced growth path. In figures 1-6 we have represented the short-run and the long-run trajectories for the ratio between physical and human capital, the levels of both physical and human capital stock, the fraction of non-leisure time devoted to goods production, the level of physical output and the level of consumption per capita. For each of the previous variables we have considered six subcases corresponding to the normal, the exogenous growth and the paradoxical cases, first when convergence happens from below $(\Omega<1)$ and, second, when convergence occurs from above $(\Omega>1)$. From now on we will refer to a process as convergent from below or above depending on the tran- 
sitional evolution experienced by the ratio between physical and human capital, which is not necessarily the case for the other inspected variables.

From these exercises, they arise some interesting patterns of temporal behavior that we would like to highlight because of their persistence and regularity. First of all, the ratio between physical and human capital shows monotonous convergence, from below or above, to a constant long-run value. The fraction of non-leisure time devoted to goods production also shows monotonous convergence to a constant long-run value, except for the exogenous growth case in which there are no transitional dynamics. Moreover, while the ratio between physical and human capital converges from below (above), the fraction of non-leisure time devoted to goods production converges from above (below) in the normal case and from below (above) in the paradoxical case. On the other hand, the dynamic patterns of the short-run adjustment for physical capital stock, physical output and consumption per capita are very similar. Convergence from below is monotonous, but short-run trajectories start showing concavity although they finish exhibiting convexity as they approach the long-run trajectories. This concave-convex feature appears less accentuated as we change from the normal to the exogenous growth and to the paradoxical case.

Instead, convergence from above is not monotonous. In this case short-run trajectories are U-shaped, decreasing at the beginning and, eventually, increasing as they approach the long-run trajectories. The U-shaped feature is more persistent across the normal, exogenous growth and paradoxical cases. Finally, the human capital stock shows a great variability in patterns of convergence, depending on whether we are in the normal, exogenous growth or paradoxical case, and according to the pattern of convergence followed by the ratio between physical and human capital. On the one hand, when the ratio physical to human capital converges from below, the short-run trajectory for human capital converges fom above (below) to the associated long-run trajectory in the normal (paradoxical) case. On the other hand, when the ratio physical to human capital converges from above, the short-run trajectory for human capital converges fom below (above) to the associated long-run trajectory in the normal (paradoxical) case. The U-shaped and concave-convex features associated with convergence from above and below, respectively, are clearly shown in the normal case, but they are less evident in the paradoxical case. In the exogenous growth case the human capital stock does not show transitional dynamics, its short-run trajectory coincides with the long-run one. 


\subsection{The effect of changes in $h_{0}$ and $K_{0}$ on the BGP levels of the variables}

In this subsection we consider the effects of a sudden increase in the initial levels of both physical and human capital stock. First of all, it is obvious that a sudden increase in $h_{0}$ will give rise to a process of convergence from below for the current levels of the variables, while a sudden increase in $K_{0}$ gives rise to a process of convergence from above. The features of these two processes of convergence for the short-run levels of the variables along the transition have been already described in a previous section. Consequently, we will concentrate on the effects of such changes on the long-run levels of the variables associated with the balanced growth path.

The expressions for the long-run values of the variables have been shown along the propositions in previous sections. However, their complete specification in terms of $h_{0}$ and $K_{0}$ requires also to solve the non-linear static system (27)-(28) for $\vartheta_{1}(0)$ and $\vartheta_{2}(0)$. This cannot be done analytically in the particular cases in which $\sigma>\beta$ (normal case) and $\sigma<\beta$ (paradoxical case). Only in the exogenous growth case in which $\sigma=\beta$, when ${ }_{2} F_{1}(0)$ and ${ }_{2} \widetilde{F}_{1}(0)$ degenerate into strictly positive constants independent of $\vartheta_{1}(0)$ and $\vartheta_{2}(0)$, we may solve in closed form the system $(27)-(28)$ for $\vartheta_{1}(0)=\left(\frac{{ }_{2} F_{1}(0) \sigma N_{0}}{K_{0}(\rho+\pi-n \sigma-\pi \sigma)}\right)^{\beta}$ and $\vartheta_{2}(0)=\left(\frac{{ }_{2} \widetilde{F}_{1}(0)(1-\beta) \sigma \epsilon N_{0}}{h_{0}(\rho-n-(\delta-\theta)(1-\sigma))(\rho+\pi-n \sigma-\pi \sigma)}\right)^{\beta}$. Hence, we obtain explicitly the initial values for the long-run levels of physical and human capital stock, physical output and consumption per capita corresponding to the balanced growth path,

$$
\begin{aligned}
& \bar{K}(0)=\frac{h_{0}(\rho-n-(\delta-\theta)(1-\sigma))}{{ }_{2} \tilde{F}_{1}(0)(1-\beta) \epsilon}\left(\frac{\epsilon}{\delta+n+\pi-\theta}\right)^{\frac{1}{1-\beta}}, \\
& \bar{h}(0)=\frac{h_{0}}{{ }_{2} \tilde{F}_{1}(0)}, \\
& \bar{Y}(0)=\frac{h_{0}(\rho-n-(\delta-\theta)(1-\sigma)) A}{{ }_{2} \tilde{F}_{1}(0)(1-\beta) \epsilon}\left(\frac{(1-\beta) \epsilon N_{0}}{\delta \beta}\right)^{1-\beta}\left(\frac{\epsilon}{\delta+n+\pi-\theta}\right)^{\frac{\beta}{1-\beta}} \text {, } \\
& \bar{c}(0)=\frac{h_{0}(\rho-n-(\delta-\theta)(1-\sigma))(\rho+\pi-n \sigma-\pi \sigma)}{{ }_{2} \tilde{F}_{1}(0)(1-\beta) \sigma \epsilon N_{0}}\left(\frac{\epsilon}{\delta+n+\pi-\theta}\right)^{\frac{1}{1-\beta}} .
\end{aligned}
$$

As we can see, these initial values (and the associated long-run trajectories) do not depend on $K_{0}$ but only on $h_{0}$. By complementing the exogenous growth case with a numerical exercise for the normal and paradoxical cases, we may conclude that after a sudden increase (decrease) in $h_{0}$ the economy will move toward a new balanced growth path 
with higher (lower) long-run levels of physical and human capital stock, physical output and consumption per capita. Instead, after a sudden increase (decrease) in $K_{0}$ only in the normal case the economy will move toward a new balanced growth path with higher (lower) long-run levels of physical and human capital stock, physical output and consumption per capita. In the paradoxical case, after a sudden increase (decrease) in $K_{0}$ the economy will move toward a new balanced growth path with lower (higher) long-run levels of physical and human capital stock, physical output and consumption per capita. In the exogenous growth case, after a sudden increase (decrease) in $K_{0}$ the economy will move to the same long-run levels of physical and human capital stock, physical output and consumption per capita, because the balanced growth path has not changed.

\section{Conclusions}

In this paper, we have shown how the use of special functions can solve one of the main difficulties arising in the study of macroeconomic dynamics, that is the indeterminacy of steady state levels in endogenous growth models. So far, authors have surmounted this major difficulty either by reducing the dimension of the involved dynamic systems or by solving the models for some special parameterizations. We argue that using some adequately chosen special functions can allow for a definitely more general, accurate and comprehensive analysis, which includes the characterization of the optimal paths of all the variables in level. We illustrate our argument on the celebrated Lucas-Uzawa model, and make clear how the chosen special functions, namely Gauss hypergeometric functions, can do the job.

Obviously, our approach is model-dependent: Gauss hypergeometric functions splendidly work for the Lucas-Uzawa model but need not work for all the non-AK endogenous models. However, the literature of special functions is so rich, so diversified and has proved so efficient and reliable in so many research areas and disciplines that it must be, in our view, a valid analytical tool in economic dynamics. While picking the convenient special functions is not always trivial, such functions, once identified, potentially allow for an inspection into the dynamics of the considered differential systems, that go far beyond the methods currently used by economists. In our view, this justifies, if not a methodological switch, at least an increasing effort to incorporate special functions in the core of economic dynamics' investigation methods. 


\section{References}

K. M. Abadir, An Introduction to Hypergeometric Functions for Economists, Econometric Reviews 18 (1999), 287-330.

M. Abramowitz and I. A. Stegun, Handbook of Mathematical Functions. New York: Dover Publications (1972).

G. Andrews, R. Askey and R. Roy, Special Functions. Cambridge: Cambridge University Press (1999).

R. Barro and X. Sala-i-Martin, Economic Growth. New-York: McGraw-Hill (1995)

W. Becken and P. Schmelcher, The Analytic Continuation of the Gaussian Hypergeometric Function ${ }_{2} F_{1}(a, b ; c ; z)$ for Arbitrary Parameters, Journal of Computational and Applied Mathematics 126 (2000), 449-478.

J. Benhabib and R. Perli, Uniqueness and Indeterminacy: On the Dynamics of Endogenous Growth, Journal of Economic Theory 63 (1994), 113-142.

E. W. Bond, P. Wang and C. K. Yip, A General Two-Sector Model of Endogenous Growth with Human and Physical Capital: Balanced Growth and Transitional Dynamics, Journal of Economic Theory 68 (1996), 149-173.

R. Boucekkine and J. R. Ruiz-Tamarit, Imbalance Effects in the Lucas Model: An Analytical Exploration. IRES DP/2004-5 (2004).

J. Caballé and M. S. Santos, On Endogenous Growth with Physical and Human Capital, Journal of Political Economy 101 (1993), 1042-1067.

M. Goursat, Sur l'équation différentielle linéaire qui admet pour intégrale la série hypergéométrique, Annales de l'Académie des Sciences et de l'École Normale Supérieure, Supplément 10 (1881), S3-S142.

E. Kummer, Uber die Hypergeometrische Reihe, J. reine angew. Math. 15 (1836), 39-83 and $127-172$.

R. E. Lucas, Jr., On the Mechanics of Economic Development, Journal of Monetary Economics 22 (1988), 3-42.

Y. Luke, The Special Functions and their Approximations. New York: Academic Press (1969).

C. B. Mulligan and X. Sala-i-Martín, Transitional Dynamics in Two Sector Models of Endogenous Growth, Quarterly Journal of Economics 108 (1993), 739-773. 
L. Rivera-Batiz and P. Romer, Economic Integration and Endogenous Growth, Quarterly Journal of Economics 106 (1991), 531-556.

N. M. Temme, Special Functions, An Introduction to the Classical Functions of Mathematical Physics. Wiley (1996).

N. M. Temme, Large Parameter Cases of the Gauss Hypergeometric Function, Journal of Computational and Applied Mathematics 153 (2003), 441-462.

D. Xie, Divergence in Economic Performance: Transitional Dynamics with Multiple Equilibria, Journal of Economic Theory 63 (1994), 97-112.

\section{Appendix}

Proof of Proposition 1 using the infinite expansion definition of the Gaussian hypergeometric function

Recall the function $Q(r)$ to integrate between 0 and $t$

$$
Q(r)=e^{\alpha C r}\left[1+A_{0} e^{(B-C) r}\right]^{\alpha},
$$

with the expressions of $A_{0}, B, C$ and $\alpha$ given in the main text. Now the integral can be written with the variable change $v=e^{\gamma r}, \gamma>0$ as

$$
\int_{0}^{t} Q(r) d r=\int_{1}^{y} v^{\frac{\alpha C}{\gamma}}\left[1+A_{0} v^{\frac{B-C}{\gamma}}\right]^{\alpha} \frac{d v}{\gamma v}
$$

with $y=e^{\gamma t}$. Choose $\gamma=\alpha C$. Then,

$$
\int_{0}^{t} Q(r) d r=\frac{1}{\alpha C} \int_{1}^{y}\left[1+A_{0} v^{\frac{B-C}{\alpha C}}\right]^{\alpha} d v .
$$

Now invoke the binomial theorem

$$
\left(1+a x^{b}\right)^{c}=\sum_{n=0}^{\infty}(-c)_{n} \frac{\left(-a x^{b}\right)^{n}}{n !}
$$

The series is convergent as long as the modulus of $a x^{b}$ is lower than one. In this circle of convergence, convergence is uniform, so that one can re-write the considered integral as

$$
\int_{0}^{t} Q(r) d r=\frac{1}{\alpha C} \sum_{n=0}^{\infty} \frac{(-\alpha)_{n}}{n !}\left(-A_{0}\right)^{n} \int_{1}^{y} v^{\frac{B-C}{\alpha C} n} d v
$$


which implies that:

$$
\int_{0}^{t} Q(r) d r=\frac{1}{\alpha C} \sum_{n=0}^{\infty} \frac{(-\alpha)_{n}}{n !}\left(-A_{0}\right)^{n} \frac{y^{1+\frac{B-C}{\alpha C} n}-1}{1+\frac{B-C}{\alpha C} n} .
$$

Now notice that for any $x, \frac{(x)_{n}}{(1+x)_{n}}=\frac{x}{x+n}$. Indeed,

$$
\frac{(x)_{n}}{(1+x)_{n}}=\frac{\Gamma(x+n)}{\Gamma(x)} \frac{\Gamma(x+1)}{\Gamma(x+1+n)},
$$

and since the gamma function has the property that: $\Gamma(x)=(x-1) \Gamma(x-1)$, we get the announced property. Therefore, we can write

$$
\frac{1}{1+\frac{B-C}{\alpha C} n}=\frac{\frac{\alpha C}{B-C}}{1+\frac{\alpha C}{B-C}}=\frac{\left(\frac{\alpha C}{B-C}\right)_{n}}{\left(1+\frac{\alpha C}{B-C}\right)_{n}}
$$

Now notice, that $a=\frac{\alpha C}{B-C}$, where $a$ is given in the statement of this proposition. Then,

$$
\int_{0}^{t} Q(r) d r=\frac{1}{\alpha C} \sum_{n=0}^{\infty} \frac{(a)_{n}(-\alpha)_{n}}{(1+a)_{n}} \frac{\left(-A_{0}\right)^{n}}{n !}\left[\frac{y y^{\frac{n}{a}}-1}{n !}\right]
$$

which yields

$$
\int_{0}^{t} Q(r) d r=\frac{1}{\alpha C}\left(y \sum_{n=0}^{\infty} \frac{(a)_{n}(-\alpha)_{n}}{(1+a)_{n}} \frac{\left(-A_{0} y^{\frac{1}{a}}\right)^{n}}{n !}-\sum_{n=0}^{\infty} \frac{(a)_{n}(-\alpha)_{n}}{(1+a)_{n}} \frac{\left(-A_{0}\right)^{n}}{n !}\right) .
$$

Using the expression of $A_{0}, B$ and $C$, and provided that $y=e^{\alpha C t}$, one obtains directly the hypergeometric representation of the capital stock given in the statement of the proposition.

\section{Proof of Lemma 2}

The $z$-function $\frac{{ }_{2} F_{1}(b, a, a+1 ; z)}{{ }_{2} F_{1}(b, a-1, a+1 ; z)}-\Omega(1-z)^{-\frac{1}{1-\beta}}$ can be differentiated using the Euler integral representations of the involved hypergeometric functions. However, the case $b<0$ is straightforward: by Lemma 1, the ratio of hypergeometric functions is decreasing and since $\Omega>0$ by condition (26), function $\Omega(1-z)^{-\frac{1}{1-\beta}}$ is increasing, implying that the difference of the two functions just above is indeed strictly decreasing. When $b>0$, things are much less obvious, and one has to resort to explicit differentiation. After some simple algebraic operations, it turns out that the sign of the derivative is determined by the sign of

$$
\Delta-\frac{a-1}{1-\beta} \Omega(1-z)^{-\frac{1}{1-\beta}-1}\left(\int_{0}^{1} t^{a-2}(1-t)(1-t z)^{-b} d t\right)^{2}
$$


where $\frac{\Delta}{b}$ is given in the proof of Lemma 1 by

$$
\int_{0}^{1} t^{a}(1-t z)^{-b-1} d t \int_{0}^{1} t^{a-2}(1-t)(1-t z)^{-b} d t-\int_{0}^{1} t^{a-1}(1-t z)^{-b} d t \int_{0}^{1} t^{a-1}(1-t)(1-t z)^{-b-1} d t .
$$

By the proof of Lemma 1, we know that $\frac{\Delta}{b}$ is always positive, therefore we cannot sign directly the difference just above. We shall proceed explicitly by introducing the double integral device as in Lemma 1 . Let $X(x, y, z)=x^{a-2}(1-x z)^{-b-1} y^{a-2}(1-y z)^{-b-1}$, with $X(x, y, z)$ positive, and symmetric in $x$ and $y$ as before. Using the same notations and exactly the same manipulations as in Lemma 1, we arrive at the conclusion that the sign of the derivative is given by the one of the double integral

$$
\int_{0}^{1} \int_{0}^{1} X(x, y, z)\left[\frac{b}{2}(x-y)^{2}-\omega^{\prime}(1-x)(1-y) \frac{(1-x z)(1-y z)}{(1-z)^{\frac{1}{1-\beta}}+1}\right] d x d y,
$$

where $\omega^{\prime}=\frac{a-1}{1-\beta} \Omega>0$. Notice that the term between brackets in the double integral is necessarily negative when $z$ tends to 1 because $\frac{1}{1-\beta}+1>2$, and the same term should be positive when $z$ tends to $-\infty$. It follows that the derivative is positive when $z$ tends $-\infty$, and negative in the neighborhood of 1 . By continuity, we deduce that there exists a z-value zeroing the derivative. We prove hereafter that such a value is unique. To this end, we demonstrate that the equation in $z$

$$
\int_{0}^{1} \int_{0}^{1} X(x, y, z) \frac{b}{2}(x-y)^{2} d x d y=\omega^{\prime} \int_{0}^{1} \int_{0}^{1} X(x, y, z)(1-x)(1-y) \frac{(1-x z)(1-y z)}{(1-z)^{\frac{1}{1-\beta}+1}} d x d y
$$

has a unique solution. Trivially the left hand side is an increasing and convex function in $z$. Differentiating the right hand side integrand, one can easily simplify the $z$-derivative into the simple expression

$$
(1+b)(1-x)(1-y) x^{a-2} y^{a-2} \frac{(1-x z)^{-b}(1-y z)^{-b}}{(1-z)^{\frac{1}{1-\beta}+2}}>0,
$$

implying that the right-hand side is also an increasing function of $z$. Trivially, the righthand side is also strictly convex because the first-order derivative is basically the inverse of the product of three positive, decreasing functions, namely, $(1-x z)^{b}(1-\mathrm{y} \mathrm{z})^{b}$, and $(1-z)^{\frac{1}{1-\beta}+2}$.

\section{Proof of Proposition 8}

Using (41), one can write the ratio $\frac{K}{N h}$ as follows:

$$
\frac{K}{h}=\frac{K_{0}}{h_{0}}\left(\frac{\vartheta_{1}(0)}{\vartheta_{2}(0)}\right)^{\frac{1}{\beta}}\left(\frac{\epsilon}{\delta+n+\pi-\theta}\right)^{\frac{1}{1-\beta}} \frac{{ }_{2} \tilde{F}_{1}(0)}{{ }_{2} F_{1}(0)} \frac{{ }_{2} F_{1}(t)}{{ }_{2} F_{1}(t)} \exp \left\{-\frac{\delta+n+\pi-\theta-\beta n}{\beta} t\right\} \beta_{0}(t),
$$


where $\beta_{0}(t)=\left(1-z^{o} \exp \left(-\frac{1-\beta}{\beta}(\delta+n+\pi-\theta) t\right)\right)^{\frac{1}{1-\beta}}, z^{o}$ being the unique solution to (33) as discussed in Section 4.3. When $b=0$, as explained in Proposition 6 , the hypergeometric functions ${ }_{2} F_{1}(t)$ and ${ }_{2} \tilde{F}_{1}(t)$ are constant. Indeed, it is trivial to show using the Euler integral representations of both that they are equal to 1 . Therefore, when $b=0$, the unique equation to (33) can be computed exactly by $1=\Omega\left(1-z^{o}\right)^{-\frac{1}{1-\beta}}$ or $z^{o}=1-\Omega^{1-\beta}$. Have also in mind that by definition, $\Omega$ is proportional to the ratio $\frac{K_{0}}{h_{0}}$.

Let us see first that the ratio $\frac{K}{N h}$ is monotonic. Under $b=0$, this ratio is proportional to function $\beta_{0}(t)$. When $\Omega>1, z^{o}<0$ and $\beta_{0}(t)$ is decreasing. When $\Omega<1,0<z^{o}<1$ and $\beta_{0}(t)$ is increasing. Thus, the ratio $\frac{K}{N h}$ is always monotonic.

Consider now the stock of physical capital with $b=0$; it is proportional to function $\xi(t)$ given by

$$
\xi(t)=\exp \left(\frac{\delta+n-\theta-\rho+n \sigma}{\sigma} t\right) \beta_{0}(t) .
$$

When $\Omega<1,0<z^{o}<1, \xi(t)$ is the product of two positive increasing functions, so it should be increasing in this case. However, if $\Omega>1, z^{o}<0$ and $\beta_{0}(t)$ is decreasing. A sufficient condition for a non-monotonic capital stock path to arise is $\xi^{\prime}(0)<0$, which amounts to

$$
(\delta+n-\theta-\rho+n \sigma)\left(1-z^{o}\right)^{\frac{1}{1-\beta}}+(\delta+n+\pi-\theta) z^{o}<0,
$$

which in turn implies, using the explicit expression of $z^{o}$ in terms of $\Omega$,

$$
\xi_{1}(\Omega)=\frac{\Omega}{\Omega^{1-\beta}-1}<\frac{\delta+n+\pi-\theta}{\delta+n-\theta-\rho+n \sigma} .
$$

To end the proof, just notice that function $\xi_{1}(\Omega)$ is a U-shaped $\Omega$-function when $\Omega>1$, with a minimum reached at $\Omega^{0}=\left(\frac{1}{\beta}\right)^{\frac{1}{1-\beta}}$. Therefore, a necessary and sufficient condition for $\xi^{\prime}(0)<0$ is $\xi_{1}\left(\Omega^{0}\right)<\frac{\delta+n+\pi-\theta}{\delta+n-\theta-\rho+n \sigma}$. In such a case, there exists a non-empty interval of $\Omega$ 's values, and therefore of the ratio $\frac{K_{0}}{h_{0}}$, which induces a non-monotonic capital stock path. 


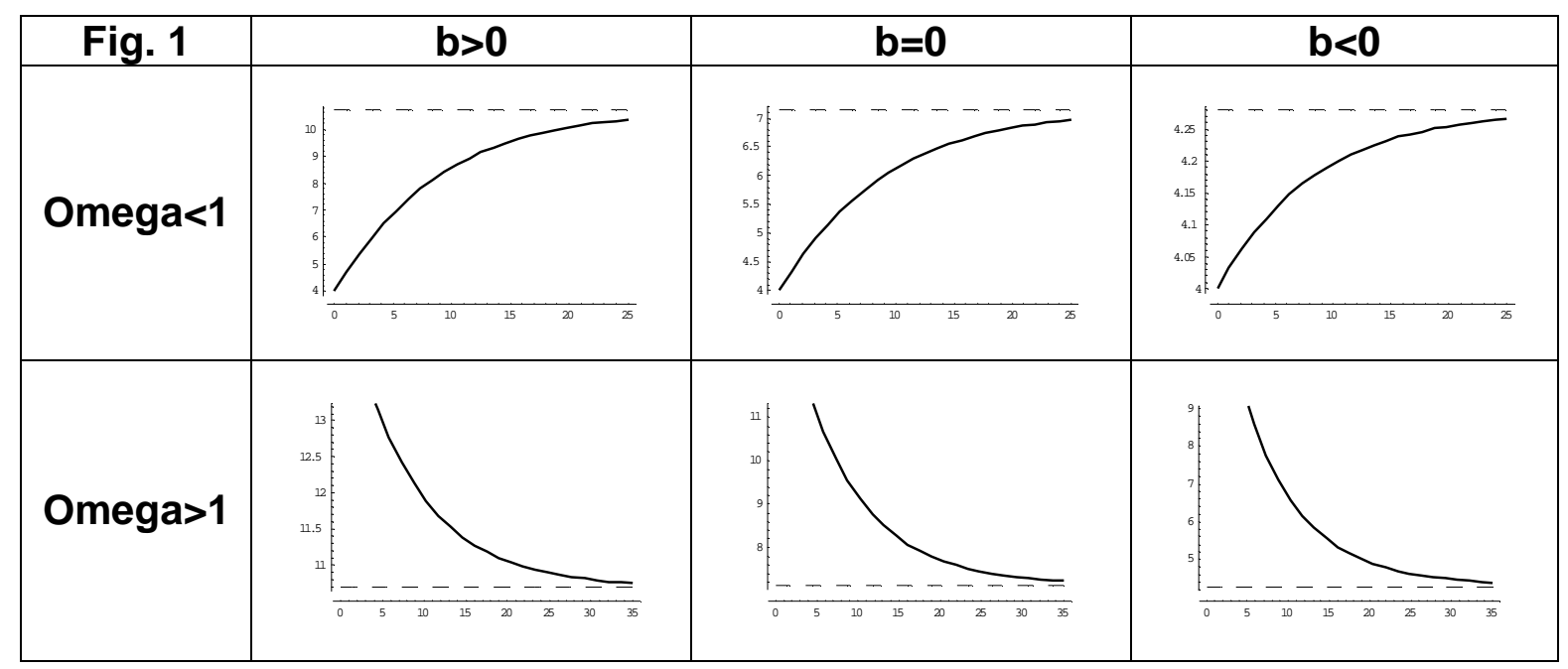

$$
\mathrm{K} / \mathrm{Nh}-\overline{\mathrm{K} / \mathrm{Nh}} \text { - - }
$$




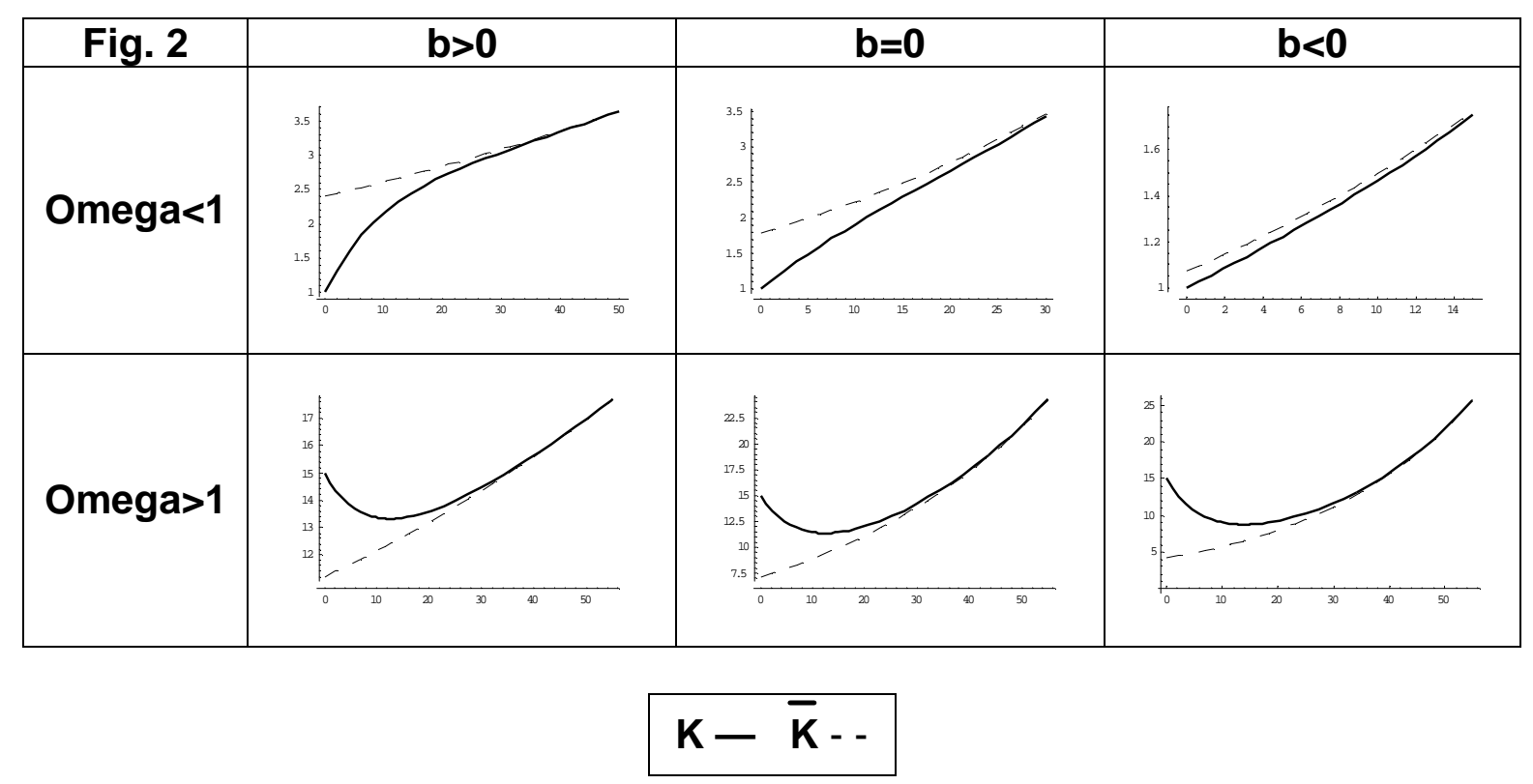




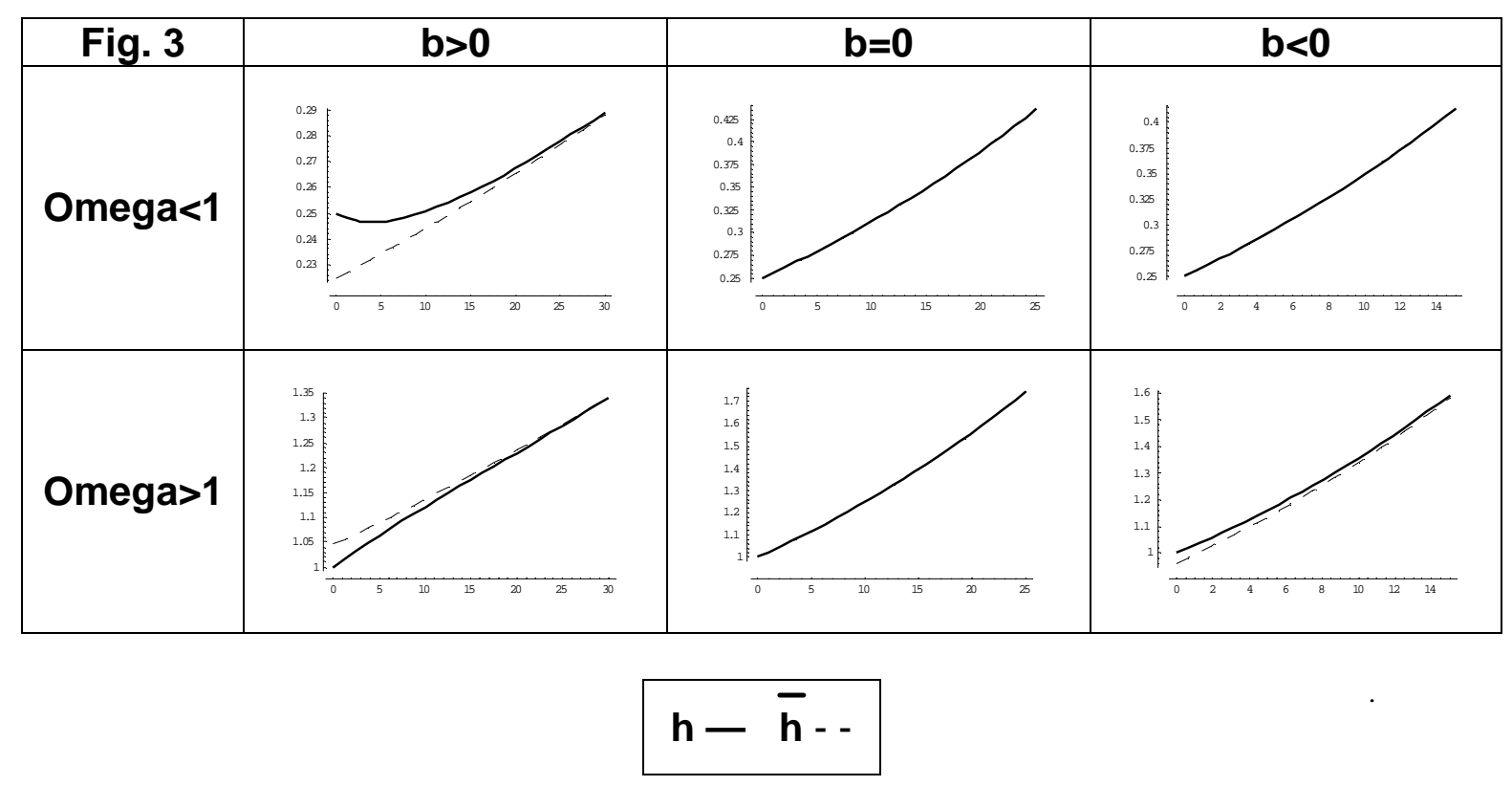




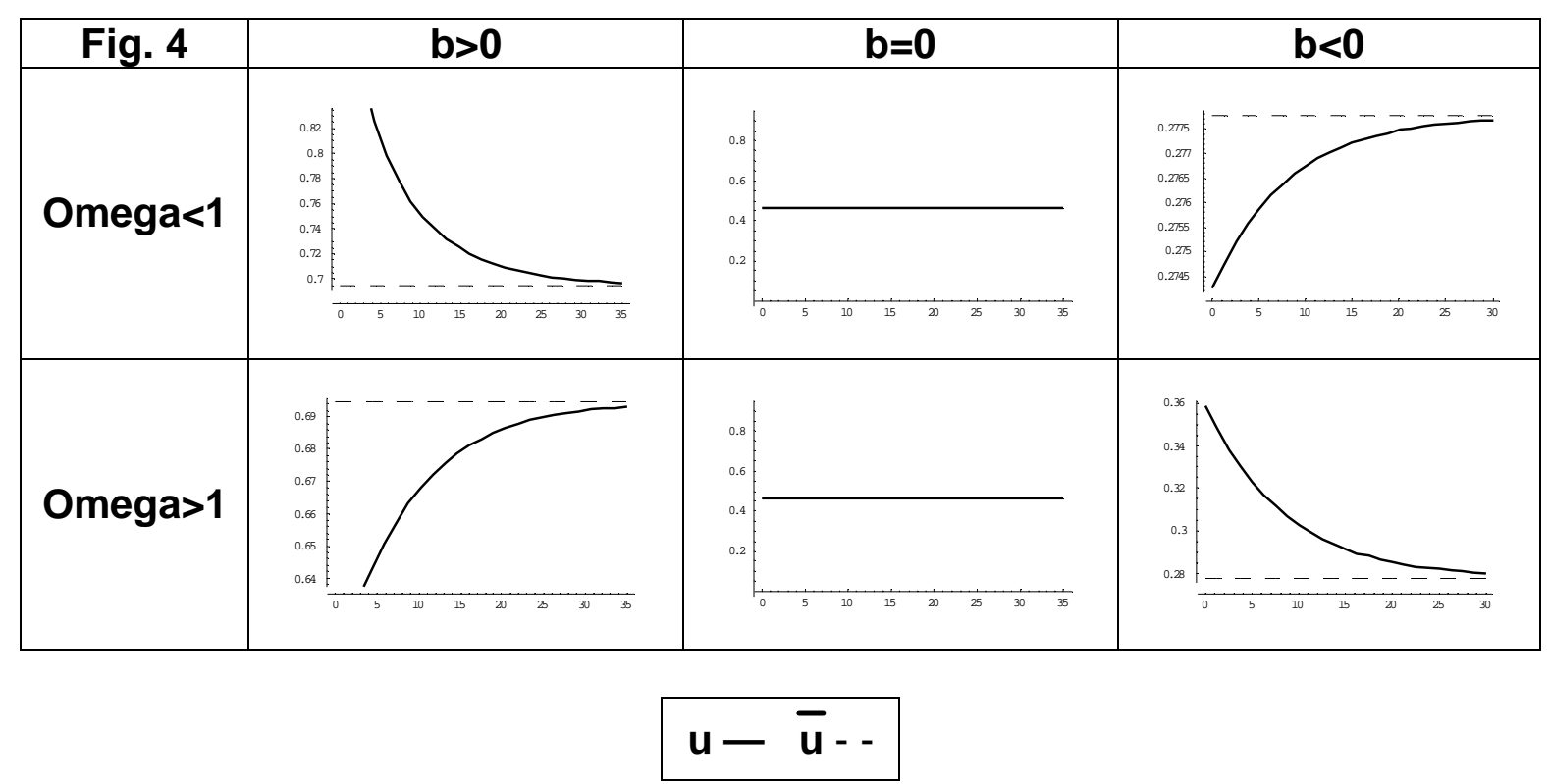




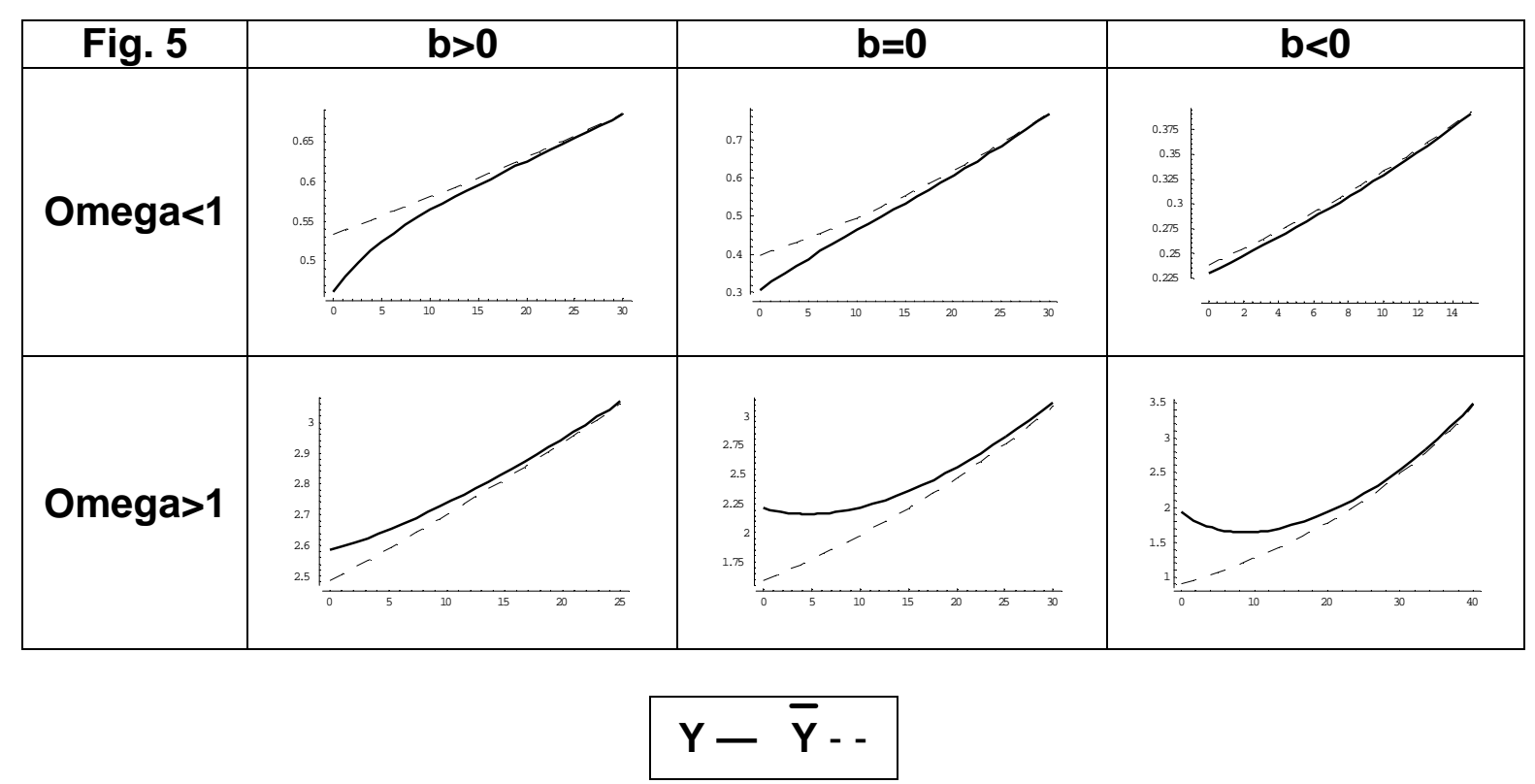




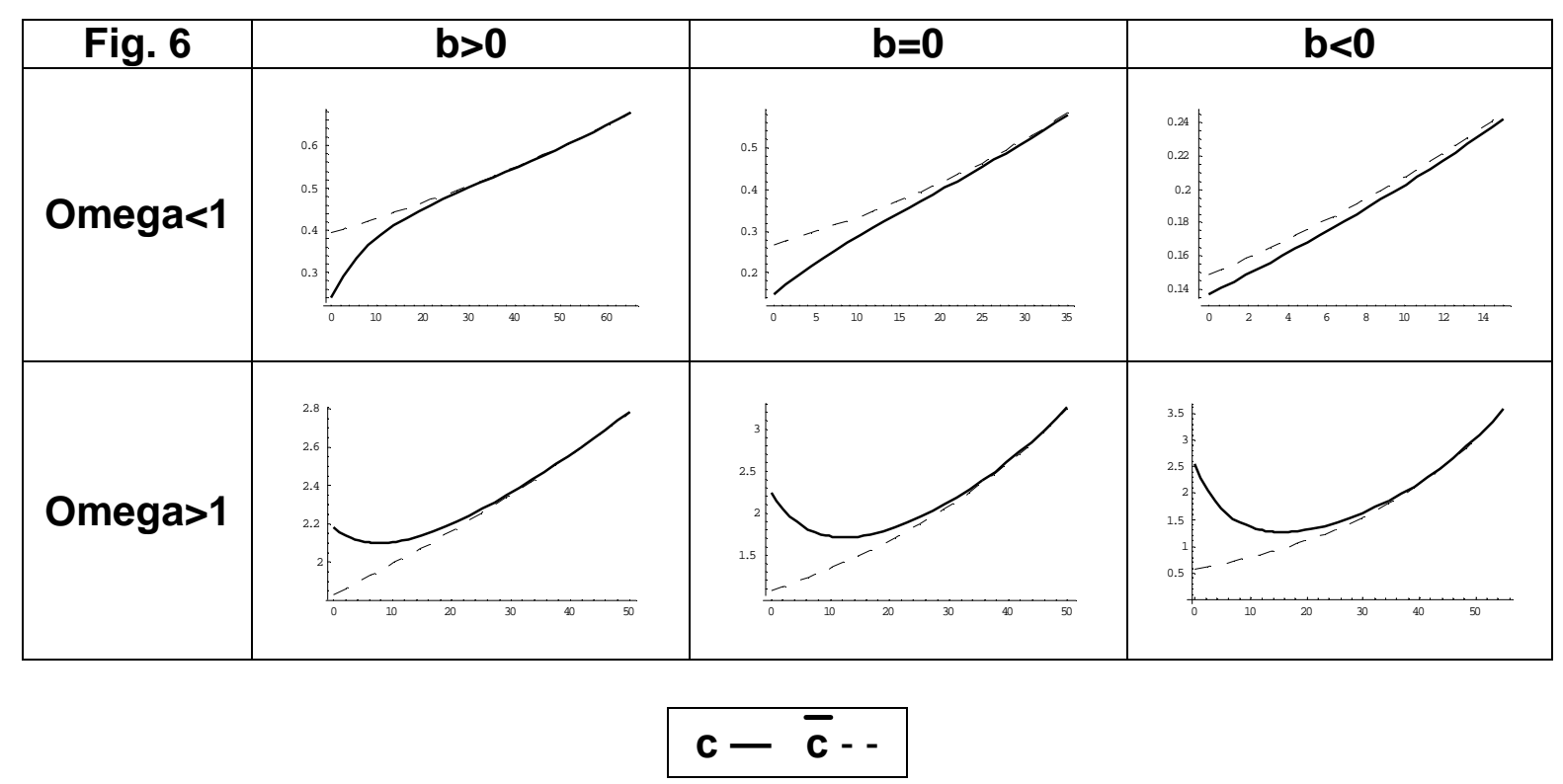

